\title{
Prediction of Biopharmaceutical Drug Disposition Classification System (BDDCS) by Structural Parameters
}

\author{
Yeganeh Golfar ${ }^{1,2}$, Ali Shayanfar ${ }^{3}$ \\ ${ }^{1}$ Biotechnology Research Center, Tabriz University of Medical Sciences, Tabriz, Iran. ${ }^{2}$ Student Research Committee \\ and Faculty of Pharmacy, Tabriz University of Medical Sciences, Tabriz, Iran. ${ }^{3 .}$ Pharmaceutical Analysis Research \\ Center and Faculty of Pharmacy, Tabriz University of Medical Sciences, Tabriz, Iran
}

Received, November 16, 2018; Revised, January 24, 2019; Accepted, June 8, 2019; Published, June 21, 2019.

\begin{abstract}
Modeling of physicochemical and pharmacokinetic properties is important for the prediction and mechanism characterization in drug discovery and development. Biopharmaceutics Drug Disposition Classification System (BDDCS) is a four-class system based on solubility and metabolism. This system is employed to delineate the role of transporters in pharmacokinetics and their interaction with metabolizing enzymes. It further anticipates drug disposition and potential drug-drug interactions in the liver and intestine. According to BDDCS, drugs are classified into four groups in terms of the extent of metabolism and solubility (high and low). In this study, structural parameters of drugs were used to develop classification-based models for the prediction of BDDCS class.

Reported BDDCS data of drugs were collected from the literature, and structural descriptors (Abraham solvation parameters and octanol-water partition coefficient $(\log \mathrm{P})$ ) were calculated by $\mathrm{ACD} / \mathrm{Labs}$ software. Data were divided into training and test sets. Classification-based models were then used to predict the class of each drug in BDDCS system using structural parameters and the validity of the established models was evaluated by an external test set.

The results of this study showed that $\log \mathrm{P}$ and Abraham solvation parameters are able to predict the class of solubility and metabolism in BDDCS system with good accuracy. Based on the developed methods for prediction solubility and metabolism class, BDDCS could be predicted in the correct with an acceptable accuracy.

Structural properties of drugs, i.e. $\log \mathrm{P}$ and Abraham solvation parameters (polarizability, hydrogen bonding acidity and basicity), are capable of estimating the class of solubility and metabolism with an acceptable accuracy.
\end{abstract}

\section{INTRODUCTION}

A major stage in drug discovery and development is evaluating the pharmacokinetic (PK) and physicochemical (PC) properties of the candidate drugs. Computational methods have become an increasingly important part of drug design and discovery over the recent decades, used for predicting the PK and PC of a candidate drug through the use of structural parameters and their correlation which is required for an efficient use of existing drugs and effective development of new drugs $[1,2]$.

The important parameters which control the rate and extent of oral drug absorption are the drug solubility and gastrointestinal permeability. The importance of these two properties has been emphasized in the biopharmaceutics classification system (BCS) that categorizes drugs into four groups based on their solubility and permeability. In this system, a drug substance is "highly soluble" when its highest dose strength is soluble in $250 \mathrm{~mL}$ or less of aqueous media over a $\mathrm{pH}$ range of 1-7.5 at $37^{\circ} \mathrm{C}$. A drug is "highly permeable" when the extent of absorption in humans is equal or greater than $90 \%$ of an administered dose [3, 4].

In 2005, Wu and Benet introduced a new system according to solubility and metabolism, to predict potential drug-drug interactions in the intestine and/or liver and drug disposition. They named this system the biopharmaceutics drug disposition classification system (BDDCS) [5]. Table 1 illustrated the BCS and BDDCS classification of drugs based on solubility and permeability/metabolism.

For class 1 drugs, only metabolic interactions need to be considered in the intestine and the liver. The efflux transporter, metabolic and the efflux transporter-enzyme interaction in the intestine must be taken into consideration for class 2 drugs.

Corresponding Author: Ali Shayanfar, Department of Pharmaceutical Chemistry, Faculty of Pharmacy, Tabriz University of Medical Sciences, Tabriz, Iran. Email: shayanfara@tbzmed.ac.ir 


\begin{tabular}{|c|c|c|c|}
\hline \multicolumn{2}{|c|}{$\begin{array}{c}\text { Class I } \\
\text { High solubility }\end{array}$} & \multicolumn{2}{|c|}{$\begin{array}{c}\text { Class II } \\
\text { Low solubility }\end{array}$} \\
\hline $\begin{array}{c}\text { BCS } \\
\text { High Permeability }\end{array}$ & $\begin{array}{c}\text { BDDCS } \\
\text { Extensive metabolism }\end{array}$ & $\begin{array}{c}\text { BCS } \\
\text { High Permeability }\end{array}$ & $\begin{array}{l}\text { BDDCS } \\
\text { Extensive metabolism }\end{array}$ \\
\hline \multicolumn{2}{|c|}{$\begin{array}{c}\text { Class III } \\
\text { High solubility }\end{array}$} & \multicolumn{2}{|c|}{$\begin{array}{c}\text { Class IV } \\
\text { Low solubility }\end{array}$} \\
\hline $\begin{array}{l}\text { BCS } \\
\text { Low Permeability }\end{array}$ & $\begin{array}{c}\text { BDDCS } \\
\text { Low metabolism }\end{array}$ & $\begin{array}{c}\text { BCS } \\
\text { Low Permeability }\end{array}$ & $\begin{array}{c}\text { BDDCS } \\
\text { Low metabolism }\end{array}$ \\
\hline
\end{tabular}

Uptake transporter, efflux transporter and uptakeefflux transporter interaction are of major importance in class 3 and 4 drugs. Therefore, the major route of elimination for class 1 and 2 drugs is metabolism, while that of class 3 and 4 drugs is renal and biliary excretion of unchanged drug [6].

The aim of BDDCS is for characterizing disposition of new molecular entities (NME) and drugs already on the market and the purpose of BCS is to render the biowaivers of in vivo bioequivalence studies facile for drugs that display no significant intestinal absorption problems [7]. Although the classification between BCS and BDDCS only differ about $5-10 \%$, Benet et al. [8] estimated the difference between BCS and BDDCS to occur for about $40 \%$ of class 1 drugs, for which FDA has granted biowaivers.

Accordingly, knowing the different BDDCS classes based on the structure of a new NME can anticipate its disposition and may reduce the cost of drug discovery and development. Various classification models have been proposed to predict the physicochemical and pharmacokinetic toxicity properties of pharmaceuticals [9-11]. Logistic regression $[12,13]$ is a simple method and an extension of linear regression and a statistical method used to estimate the relationship between the independent variables in modeling the binary response data.

The purpose of the present research was to predict the class of a drug in BDDCS system. Therefore, the structural parameters of drugs and classification-based models were used to predict solubility and metabolism classes (high or low), separately. Then, these models were applied to estimate BDDCS class.

\section{COMPUTATIONAL METHODS}

Processing metabolism and solubility data and calculation of molecular descriptors

Data and molecular descriptors

Experimental classification of 927 drugs based on BDDCS (metabolism and solubility) and corresponding maximum dose of each compound were taken from the published data set of Benet and coworkers [8]. A data set of 595 orally administered drugs from which their highest dose strength expressed as a mass quantity, not as a concentration (e.g. solutions), were included for metabolism and solubility modeling following the removal of all salt forms. For all 595 compounds, SMILES (simplified molecular-input line-entry system) code was employed via www.pubchem.ncbi.nlm.nih.gov, and the numerical values for $\operatorname{clog} \mathrm{P}$ (calculated octanolwater partition coefficient) and Abraham solvation parameters were computed by ACD/Labs software (https://ilab.acdlabs.com). These parameters are independent variables, or descriptors, with the following solute properties: $\mathrm{E}$ is the excess molar refraction, $\mathrm{S}$ indicates dipolarity/polarizability descriptors of the solute, $\mathrm{A}$ and $\mathrm{B}$ are the solute hydrogen-bond acidity and basicity, and V is the McGowan volume of the solute [14].

\section{Classification of data to training and test sets}

Dataset was sorted based on ascending clogP, where from six consecutive compounds, one was allocated to the test set. The training sets were used to build separate models and predict metabolism and solubility classes. Therefore, 595 compounds classified into 496 compounds for the training set and the remaining data (99 data points) as test set to evaluate the prediction capability of the developed models.

\section{Binning of solubility compounds}

496 compounds were divided into the following groups:

1) Compounds with high solubility (class 1 and 3) according to BDDCS as class $\mathrm{H}$ (high solubility).

2) Compounds with low solubility (class 2 and 4) according BDDCS as class L (low solubility).

\section{Binning of metabolism compounds}

496 compounds were divided into the following groups:

1) Compounds with high metabolism (class 1 and 2) according to BDDCS as class $\mathrm{H}$ (high metabolism). 
2) Compounds with low metabolism (class 3 and 4) according to reference BDDCS as class L (low metabolism).

\section{Modeling}

Various thresholds and models developed by the training set (based on the structural parameters, clogP and Abraham solvation parameters) were analyzed, and the optimal parameters and their values were obtained by calculating the prediction accuracy of solubility and metabolism class (number of correct prediction/total data) for the test set. The threshold to define the boundary between high and low metabolism was set at $\operatorname{cog} \mathrm{P}=2$. Therefore, a compound with $\operatorname{cog} \mathrm{P}>2$ would be defined as high metabolism and a compound with $\operatorname{cog} \mathrm{P}<2$ would follow a binary regression model. The definition of the borderline between high and low solubility was set at maximum dose $=10 \mathrm{mg}$, hence compounds with a maximum dose under $10 \mathrm{mg}$ would be defined as highly soluble and those with a maximum dose higher than $10 \mathrm{mg}$ followed a binary logistic regression by SPSS version 21 software (www.spss.com.hk) for classification of drugs into the two mentioned sub-groups.

For the binary regression method, each class (metabolism and solubility) was set as a dependent variable and binary classification was carried out using selected molecular descriptors as independent variables to develop the models for metabolism and solubility. Features were selected for the logistic regression model based on probability values (p-value) associated with each descriptor whenever they were statistically significant at the $99 \%$ level $(p<0.01)$. It shows the probability that the descriptor is there by chance is less than $1 \%$ [15].

$\mathrm{P}$-values and coefficients in regression analysis work together to show relationships in the model that are statistically significant. The software compares the t-statistic with values in the Student's $\mathrm{t}$ distribution to determine the $\mathrm{p}$-value.

The models were developed by binary logistic regression and structural parameters, $\operatorname{cog} \mathrm{P}$ and Abraham solvation parameters, and maximum dose to predict BDDCS class. Separate models for metabolism and solubility were built using training sets of 496 compounds. The prediction capability of the developed models was checked by a test set composed of 99 compounds.

\section{RESULTS}

\section{Solubility prediction}

In the training set, $20 \%$ of the drugs had a maximum dose lower than $10(\mathrm{~N}=100)$. Most of these drugs ( $85 \%$ ) belonged to class 1 and 3 (highly soluble), a criterion applied to classify them in the correct group with a good accuracy. Based on the definition, when the highest dose strength of a drug substance is soluble in $250 \mathrm{~mL}$ or less of aqueous media over a $\mathrm{pH}$ range of $1-7.5$ at $37^{\circ} \mathrm{C}$, it is considered as "highly soluble"[16]. Therefore, dose is a critical parameter based on the obtained results in this work and most drugs with a maximum dose of $10 \mathrm{mg}$ or less are highly soluble. The remaining data points $(\mathrm{N}=396)$ were applied to develop a model by Abraham solvation parameters and clogP based on binary logistic regression, where the obtained model is:

$$
P=\frac{e^{(3.203+1.295 B-.882 S-0.373 \operatorname{clog} P)}}{1+e^{(3.203+1.295 B-.882 S-0.373 \operatorname{clog} P)}} \quad \text { Eq. } 1
$$

where $\mathrm{P}$ is the probability of binary responses (class 0 or 1) based on the solubility. In addition, probability values ( $p$-value) associated with each descriptor were less than 0.01 . The model was able to predict (Eq. 1) $79 \%$ and $68 \%$ of high and low soluble drugs of the training set in the correct group, respectively. Overall, using maximum dose, $\mathrm{B}$ (hydrogen bond basicity), S (polarizability) and clogP, the solubility class of $74 \%$ of compounds was classified in the correct group.

To evaluate the prediction capability of the model, 99 compounds in test set were used to predict the correct class of drug based on solubility. $82 \%$ of the compounds with maximum doses lower than $10(\mathrm{~N}=17)$ were highly soluble, and Eq. 1 could accurately predict $73 \%$ of drugs with maximum doses higher than $10(\mathrm{~N}=82)$ in correct group. Therefore, total prediction accuracy for the test set was $74 \%$.

\section{Metabolism prediction}

In training set, $54 \%$ drugs had a clogP higher than $2(\mathrm{~N}=270)$. Most of these drugs $(91 \%)$ belonged to class 1 and 2 (high metabolism), a criterion applied to classify them in the correct group with a good accuracy. The remaining data points $(\mathrm{N}=226)$ were applied to develop a model by Abraham solvation parameters and $\operatorname{cog} \mathrm{P}$ based on binary logistic regression, where the obtained model is:

$$
p=\frac{e^{(0.734-1.260 A+.357 \operatorname{clog} P)}}{1+e^{(0.734-1.260 A+.357 \operatorname{clog} P)}} \quad \text { Eq. } 2
$$

Eq. 2 was able to correctly predict $80 \%$ and $66 \%$ of high and low metabolize drugs in the correct class, respectively. Generally, using A (hydrogen bond acidity) and clogP, metabolism class of $83 \%$ 
of the studied drugs could be classified in the correct group.

To evaluate the prediction capability of the model, 99 compounds in the test set were used to predict the correct class of drug based on metabolism. $89 \%$ of compounds with clogP higher than $2(\mathrm{~N}=54)$ were high metabolism and Eq. 2 could accurately predict $84 \%$ of drugs with clogP lower than $2(\mathrm{~N}=45)$ in the correct group. Overall, total prediction accuracy for the test set was $86 \%$.

\section{BDDCS prediction}

The results associated with the prediction of the BDDCS class of the studied drugs are shown in Table 2. Based on the developed methods for prediction solubility and metabolism class, $64 \%$ and $63 \%$ of training and test set could be predicted in the correct BDDCS class.

\section{DISCUSSION}

In this study, we described a computational method to predict the BDDCS class of compounds based on their molecular descriptors. The dataset was obtained from the published data set of Benet et al. [8]. Due to the removing salts and the solution forms of drugs, the dataset was reduced to 595 oral drugs, divided into training (496 drugs) and test (99 drugs) sets. As outlined earlier, BDDCS is a modification of the BCS [17] that utilizes drug metabolism rather than intestinal permeability [18]. In this work, we attempted to build models to predict the class of metabolism and solubility class.

Both metabolism and solubility are important properties in drug discovery. However, these properties are complex and can be difficult to model. BDDCS class prediction can overcome variable metabolism and solubility data by predicting the compound classes rather than specific values as a primary initial screening of compounds. However, suitable thresholds for discriminating between high and low metabolism/solubility should be carefully considered.

There are many factors influencing solubility and metabolism, where a threshold for discriminating between high and low metabolism/solubility can be used to improve the prediction of BDDCS class. The definition of the borderline between high and low solubility is set at maximum dose $10 \mathrm{mg}$. Compounds with maximum doses lower than $10 \mathrm{mg}$ were defined as highly soluble ( $85 \%$ and $82 \%$ for training and test set, respectively) while only $55 \%$ of drugs with maximum dose $>10$ are high soluble. Therefore, Eq. 1 is necessary for classification of data with a high dose in correct group. Using B, S and clogP (Eq. 1), solubility class could be accurately predicted $(74 \%$ and $73 \%$ for training and test set, respectively) for those with maximum doses of higher than 10. The results for the prediction solubility class of drugs are shown in Figure 1. These data show that maximum dose is necessary for prediction class of solubility.

The optimal threshold to define the boundary between high and low metabolism based on the training set is set at $\operatorname{cog} \mathrm{P}=2$. In compounds with clogP higher than 2, metabolism classes were predicted with good accuracy (91\% and $89 \%$ for training and test set, respectively). In other words, the compounds with $\operatorname{cog} \mathrm{P}>2$ are lipophilic compounds with high metabolism. Moreover, the model developed based on logistic regression by clog P and A (Eq. 2) for low metabolism drugs $(\operatorname{cog} \mathrm{P}<2)$ was able to predict the metabolism class of studied compounds with an acceptable accuracy $(73 \%$ and $84 \%$ for training and test set, respectively). These findings indicate that $\operatorname{cog} \mathrm{P}$ which used to predict various physicochemical, pharmacokinetic and biological properties of compounds [19-21] and could be calculated based on the structure of compounds with good accuracy [22], is a crucial parameter to predicting the metabolism class of drugs in BDDCS. The intrinsic lipophilicity $(\log \mathrm{P})$ is a common parameter for predicting solubility and metabolism. It is a physical feature introduced to describe a compound's affinity towards lipid-like environments, affecting drug absorption, bioavailability, hydrophobic drug-receptor interactions, and metabolism of molecules. It describes the equilibrium distribution of molecular drug candidates (unionized form of the molecule) between water and octanol and is independent of $\mathrm{pH}$. Several researchers have reported an inverse relationship between clogP and aqueous solubility [23-25]. ClogP has been utilized instead of experimental $\log \mathrm{P}$ in modeling studies, where there is a high correlation between them [26, 27]. A (hydrogen bond acidity) is yet another significant parameter for predicting the metabolism of low lipophilic drugs $\operatorname{cog} \mathrm{P}<2$. The results related to the prediction metabolism class of the studied drugs were shown in Figure 2.

Collinear descriptors $\left(\mathrm{R}^{2}>0.8\right)$ should be avoided in developing models [28], as they may entail the over fitting of the data. The intercorrelation between the selected parameters in Eq. 1 and 2 was less than 0.5 , a value corroborating the validity of the developed model from this viewpoint. 


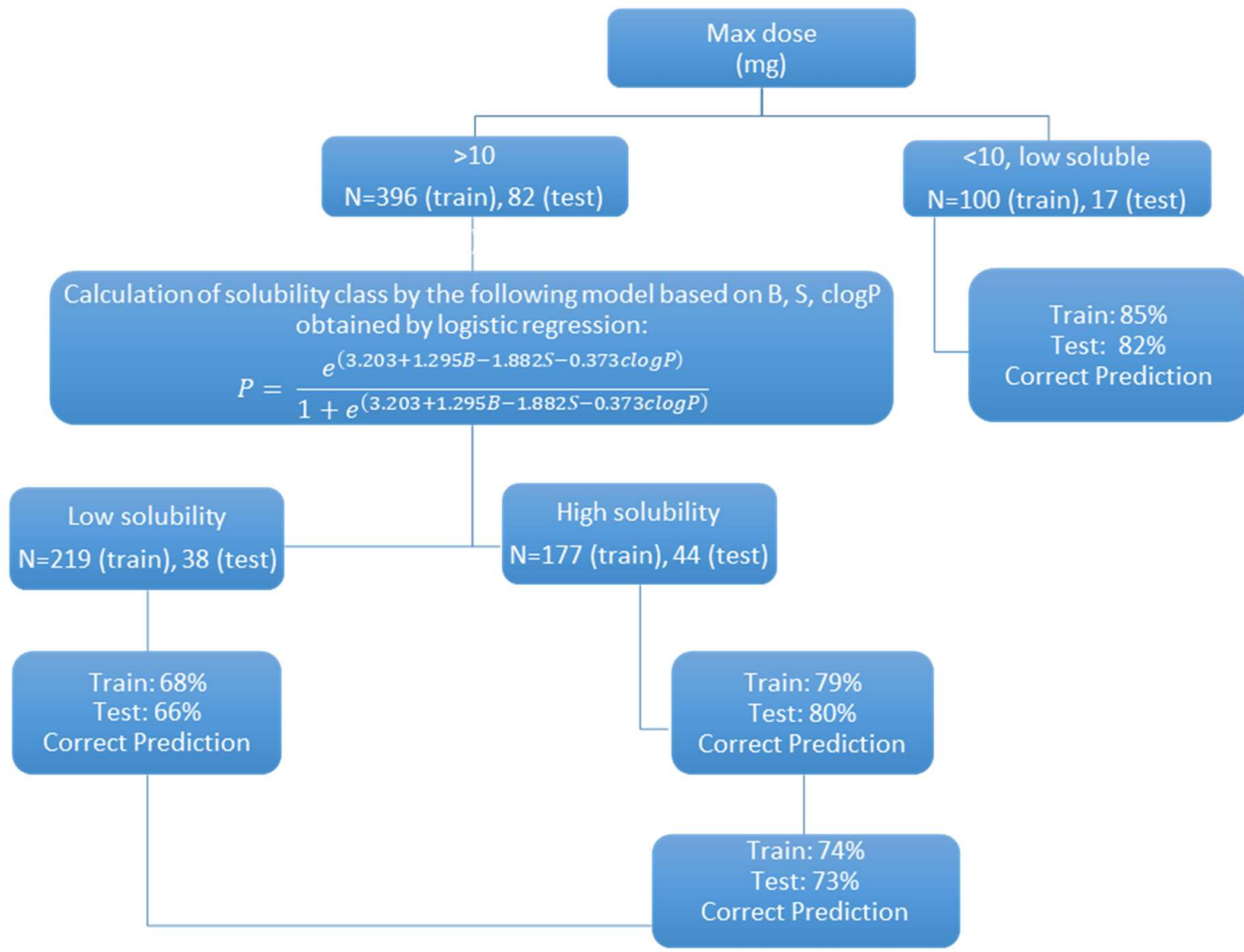

Figure 1. Prediction of solubility class. Number of training set and test sets are 496 and 99, respectively. N is number of compounds.

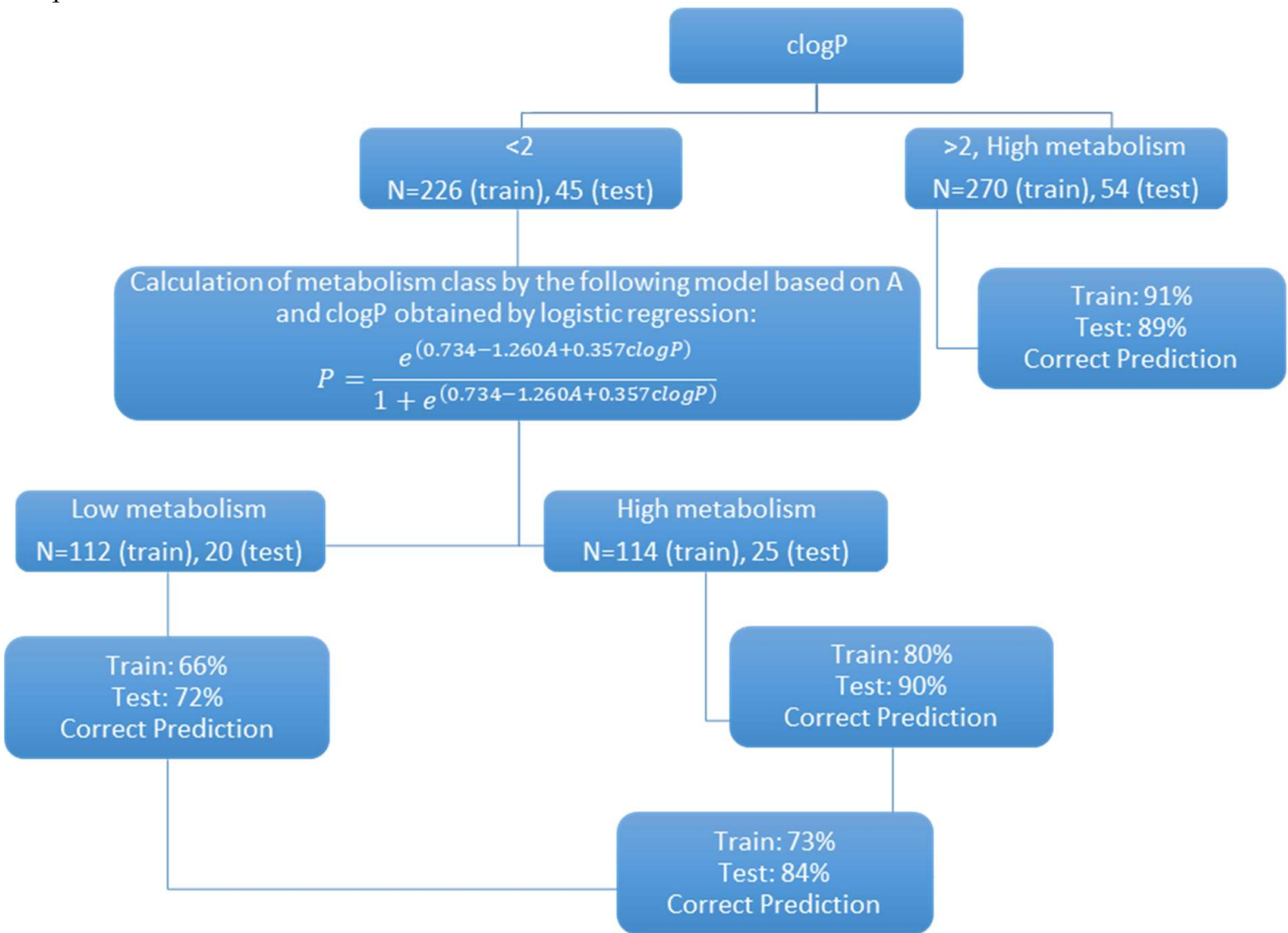

Figure 2. Prediction of metabolism class. Number of training set and test sets are 496 and 99 , respectively. $\mathrm{N}$ is number of compounds. 
Similar results associated with the prediction of the solubility and metabolism class of external test set confirm the prediction capability of the developed models.

Both models applied the Abraham solvation parameters for the prediction of solubility and metabolism, confirming the previous studies which applied these parameters for the analysis and prediction of physicochemical properties and pharmacokinetic parameters such as adsorption, distribution and toxicological features of drugs $[14,29,30]$.

A computational procedure for predicting the BDDCS class was attempted by Broccatelli et al [31] by molecular structures calculated from the VolSurft software. Similarly, the proposed method predicted the BDDCS class with relatively good accuracy with a general lack of predictability for class 4 drugs. However, relatively simple statistical method (logistic regression) and descriptors i.e. clog $\mathrm{P}$ and Abraham solvation parameters of solute, are more acceptable in modeling studies [15], and could be useful in predicting solubility and metabolism class and estimating drug-drug interactions and transporter effects in drug disposition.

Solubility and metabolism are complex parameters whose values are affected by various factors. It is possible that the applied parameters were not sufficient to estimate the correct class. However, the variations and inaccuracy of data are among the possible reasons for the unsuccessful attempts of medicinal chemists in developing models with high capability of predicting the physicochemical, pharmacokinetic and activity of drugs. For instance, the best model for aqueous solubility prediction has a mean percentage deviation (MPD) of more than $100 \%$ [32], and the MPD value for solubility in the solvent mixture is higher than $25 \%$ for pharmaceuticals [33].

In this study, we demonstrated that the developed models for prediction solubility and metabolism could estimate BDDCS with $64 \%$ accuracy, a value which does not seem to be very satisfactory. However, following the publication of BDDCS for over 900 data points in 2011 [8], Benet and coworkers [34] in 2016 amend the classification of 13 drugs. In this data set, five compounds in the training set (colchicine, diclofenac, flecainide, pindolol and saxagliptin) and four compounds in the test set (aliskiren, clonidine, metoclopramide and pitavastatin) were corrected in terms of BDDCS. These data (old and new class, and prediction class in this study) are listed in Table 3. According to the old data set, the proposed method can predict only one compound in the correct group, while based on the updated data, the BDDCS class of five data points (diclofenac, flecainide, metoclopramide. pindolol and clonidine) was classified in the correct group. Given the possible errors in experimental data and maximum $25 \%$ prediction based on probability rules, the obtained results confirm the good accuracy of the developed models. Given the possible incorrect errors in experimental data and maximum $25 \%$ prediction based on probability rules, the obtained results confirm the good accuracy of the developed models.

\section{CONCLUSION}

To predict the BDDCS of compounds, we proposed the use of threshold values by two parameters, clogP for metabolism and maximum dose for solubility, and logistic regression based models using clogP and Abraham solvation parameters.

The descriptors utilized in this work (the three Abraham solvation parameters, namely A, B and S) showing hydrogen bond acidity and basicity, and polarizability, respectively, $\operatorname{cog} \mathrm{P}$ and maximum dose of compounds are adequate for the prediction of solubility and metabolism, and can be used in the prediction of BDDCS of drugs with an acceptable accuracy.

\section{ACKNOWLEDGMENT}

This article is a part of the results of Y.G's Pharm.D thesis No. 3986 registered at Faculty of Pharmacy, Tabriz University of Medical Sciences, Tabriz, Iran.

\section{REFERENCES}

1. Stepensky D. Prediction of drug disposition on the basis of its chemical structure. Clin Pharmacokinet. 2013;52(6):415-31.

2. Dearden JC. Whither QSAR? Pharm Sci. 2017;23(2):82-3.

3. Amidon GL, Lennernäs H, Shah VP, Crison JR. A theoretical basis for a biopharmaceutic drug classification: the correlation of in vitro drug product dissolution and in vivo bioavailability. Pharm Res. 1995;12(3):413-20.

4. Yu LX, Amidon GL, Polli JE, Zhao H, Mehta MU, Conner DP, et al. Biopharmaceutics classification system: The scientific basis for biowaiver extensions. Pharm Res. 2002;19(7):921-5.

5. Wu CY, Benet LZ. Predicting drug disposition via application of BCS: transport/absorption/elimination interplay and development of a biopharmaceutics drug 
disposition classification system. Pharm Res. 2005;22(1):11-23.

6. Benet LZ. Predicting drug disposition via application of a biopharmaceutics drug disposition classification system. Basic Clin Pharmacol Toxicol. 2010;106(3):162-7.

7. Benet LZ. The role of BCS (biopharmaceutics classification system) and BDDCS (biopharmaceutics drug disposition classification system) in drug development. J Pharm Sci. 2013;102(1):34-42.

8. Benet LZ, Broccatelli F, Oprea TI. BDDCS applied to over 900 drugs. AAPS J. 2011;13(4):519-47.

9. Newby D, Freitas AA, Ghafourian T. Comparing multilabel classification methods for provisional biopharmaceutics class prediction. Mol Pharm. 2015;12(1):87-102.

10. Toropova AP, Toropov AA. CORAL: Binary classifications (active/inactive) for drug-induced liver injury. Toxicol Lett. 2017;268:51-7.

11. Dave RA, Morris ME. Novel high/low solubility classification methods for new molecular entities. Int J Pharm. 2016;511(1):111-26.

12. Hosmer Jr DW, Lemeshow S, Sturdivant RX. Applied logistic regression: John Wiley \& Sons; NewYork, 2013.

13. Ren YY, Zhou LC, Yang L, Liu PY, Zhao BW, Liu HX. Predicting the aquatic toxicity mode of action using logistic regression and linear discriminant analysis. SAR QSAR Environ Res. 2016;27(9):721-46.

14. Acree WE, Grubbs LM, Abraham MH. Prediction of partition coefficients and permeability of drug molecules in biological systems with Abraham model solute descriptors derived from measured solubilities and water-to-organic solvent partition coefficients. In: Acree WE. (ed.) Toxicity and Drug Testing, InTech Publisher; New York, 2012, pp. 91-128.

15. Dearden JC, Cronin MTD, Kaiser KLE. How not to develop a quantitative structure-activity or structure-property relationship (QSAR/QSPR). SAR QSAR Environ Res. 2009;20(3-4):241-66.

16. Chen ML, Amidon GL, Benet LZ, Lennernas H, Yu LX. The BCS, BDDCS, and regulatory guidances. Pharm Res. 2011;28(7):1774-8.

17. Emami J. In vitro-in vivo correlation: from theory to applications. J Pharm Pharm Sci. 2006;9(2):16989.

18. Larregieu CA, Benet LZ. Distinguishing between the permeability relationships with absorption and metabolism to improve BCS and BDDCS predictions in early drug discovery. Mol Pharm. 2014;11(4):1335-44.

19. Smith CJ, Perfetti TA, Ko GM, Garg R. Ames mutagenicity, structural alerts of carcinogenicity, Hansch QSAR parameters (ClogP, CMR, MgVol), tumor site concordance/multiplicity, and tumorigenicity rank in NTP 2-year rodent studies. Toxicol Res Appl. 2018;2:2397847318759327.
20. Emami S, Jouyban A, Valizadeh H, Shayanfar A. Are Crystallinity Parameters Critical for Drug Solubility Prediction? J Solut Chem. 2015;44(12):2297-315.

21. Ghafourian T, Amin Z. QSAR models for the prediction of plasma protein binding. BioImpacts. 2013;3(1):21.

22. Hughes LD, Palmer DS, Nigsch F, Mitchell JBO. Why are some properties more difficult to predict than others? A study of QSPR models of solubility, melting point, and $\log \mathrm{P}$. J Chem Inf Model. 2008;48(1):220-32.

23. Faller B, Ertl P. Computational approaches to determine drug solubility. Adv Drug deliv Rev. 2007;59(7):533-45.

24. Salahinejad M, Le TC, Winkler DA. Aqueous solubility prediction: Do crystal lattice interactions help? Mol Pharm. 2013;10(7):2757-66.

25. Louis B, Agrawal VK, Khadikar PV. Prediction of intrinsic solubility of generic drugs using MLR, ANN and SVM analyses. Eur J Med Chem. 2010;45(9):4018-25.

26. Machatha SG, Yalkowsky SH. Comparison of the octanol/water partition coefficients calculated by $\mathrm{C} \log \mathrm{P} \AA, \quad \mathrm{ACD} \log \mathrm{P}$ and $\quad \operatorname{KowWin} \AA$ to experimentally determined values. Int $\mathrm{J}$ Pharm. 2005;294(1-2):185-92.

27. Jouyban A. Solubility prediction of drugs in waterpolyethylene glycol 400 mixtures using JouybanAcree model. Chem Pharm Bull. 2006;54(11):1561-6.

28. Cherkasov A, Muratov EN, Fourches D, Varnek A, Baskin II, Cronin M, et al. QSAR modeling: Where have you been? Where are you going to? J Med Chem. 2014;57(12):4977-5010.

29. Abraham MH. Human intestinal absorption Neutral molecules and ionic species. J Pharm Sci. 2014;103(7):1956-66.

30. Abraham MH, Smith RE, Luchtefeld R, Boorem AJ, Lou R, Acree Jr WE. Prediction of solubility of drugs and other compounds in organic solvents. J Pharm Sci. 2010;99(3):1500-15.

31. Broccatelli F, Cruciani G, Benet LZ, Oprea TI. BDDCS class prediction for new molecular entities. Mol Pharm. 2012;9(3):570-80.

32. Shayanfar A, Fakhree M, Jouyban A. A simple QSPR model to predict aqueous solubility of drugs. J Drug Deliv Sci Tec. 2010;20(6):467-76.

33. Jouyban A. Review of the cosolvency models for predicting solubility of drugs in water-cosolvent mixtures. J Pharm Pharm Sci. 2008;11(1):32-58.

34. Hosey CM, Chan R, Benet LZ. BDDCS Predictions, Self-Correcting aspects of BDDCS assignments, BDDCS assignment Corrections, and classification for more than 175 additional drugs. AAPS J. 2016;18(1):251-60. 
J Pharm Pharm Sci (www.cspsCanada.org) 22, 247 - 269, 2019

Table 2. List of studied compounds, BDDCS class, structural parameters and prediction class of solubility (high=H, low=L), metabolism (high=H, low=L) and BDDCS class by the developed methods in this study (A: hydrogen bond acidity, B: hydrogen bond basicity, S: polarizability, log P: octanol-water partition coefficient

\begin{tabular}{|c|c|c|c|c|c|c|c|c|c|c|}
\hline No. & Generic Name & BDDCS & A: & B: & S: & $\operatorname{clog} P$ & $\begin{array}{l}\text { Dose } \\
\text { (mg) }\end{array}$ & $\begin{array}{l}\text { Prediction of } \\
\text { solubility }\end{array}$ & $\begin{array}{l}\text { Prediction of } \\
\text { metabolism }\end{array}$ & $\begin{array}{l}\text { Prediction of } \\
\text { BDDCS }\end{array}$ \\
\hline \multicolumn{11}{|c|}{ Training set } \\
\hline 1 & Acarbose & 1 & 3.35 & 6.22 & 4.52 & -6.66 & 100 & $\mathrm{H}$ & $\mathrm{L}$ & 3 \\
\hline 2 & Acetaminophen & 1 & 0.95 & 0.8 & 1.63 & 0.49 & 1000 & $\mathrm{H}$ & $\mathrm{L}$ & 3 \\
\hline 3 & Acetazolamide & 4 & 0.85 & 1.5 & 2.55 & -0.98 & 250 & $\mathrm{H}$ & $\mathrm{L}$ & 3 \\
\hline 4 & Acetohexamide & 1 & 0.59 & 1.46 & 2.79 & 2.25 & 500 & $\mathrm{~L}$ & $\mathrm{H}$ & 2 \\
\hline 5 & Acrivastine & 3 & 0.57 & 1.45 & 2 & 1.46 & 8 & $\mathrm{H}$ & $\mathrm{H}$ & 1 \\
\hline 6 & Acyclovir & 4 & 0.65 & 2.18 & 1.95 & -2.42 & 800 & $\mathrm{H}$ & $\mathrm{L}$ & 3 \\
\hline 7 & Adefovir Dipivoxil & 3 & 0.23 & 2.91 & 2.93 & -1.98 & 10 & $\mathrm{H}$ & $\mathrm{L}$ & 3 \\
\hline 8 & Albendazole & 2 & 0.71 & 1.12 & 1.96 & 3.46 & 200 & $\mathrm{~L}$ & $\mathrm{H}$ & 2 \\
\hline 9 & Albuterol & 3 & 1.19 & 1.82 & 1.26 & 0.06 & 4 & $\mathrm{H}$ & $\mathrm{L}$ & 3 \\
\hline 10 & Alfacalcidol & 1 & 0.63 & 1.01 & 1.01 & 8.24 & 1 & $\mathrm{H}$ & $\mathrm{H}$ & 1 \\
\hline 11 & Allopurinol & 2 & 0.27 & 1.54 & 1.04 & 0.63 & 300 & $\mathrm{H}$ & $\mathrm{H}$ & 1 \\
\hline 12 & Almotriptan & 3 & 0.31 & 1.65 & 2.16 & 1.79 & 12.5 & $\mathrm{H}$ & $\mathrm{H}$ & 1 \\
\hline 13 & Alosetron & 1 & 0.35 & 1.38 & 2.64 & 1.74 & 1 & $\mathrm{H}$ & $\mathrm{H}$ & 1 \\
\hline 14 & Alprazolam & 1 & 0 & 0.84 & 1.95 & 2.56 & 2 & $\mathrm{H}$ & $\mathrm{H}$ & 1 \\
\hline 15 & Alprenolol & 1 & 0.29 & 1.36 & 1.12 & -0.86 & 200 & $\mathrm{H}$ & $\mathrm{H}$ & 1 \\
\hline 16 & Altretamine & 2 & 0 & 1.3 & 1.53 & 1.67 & 10 & $\mathrm{H}$ & $\mathrm{H}$ & 1 \\
\hline 17 & Alvimopan & 3 & 1.33 & 1.96 & 1.41 & 2.16 & 100 & $\mathrm{H}$ & $\mathrm{H}$ & 1 \\
\hline 18 & Amantadine & 3 & 0.21 & 0.64 & 0.68 & 2 & 100 & $\mathrm{H}$ & $\mathrm{H}$ & 1 \\
\hline 19 & Ambrisentan & 1 & 0.57 & 1.52 & 2.32 & 3.33 & 10 & $\mathrm{~L}$ & $\mathrm{H}$ & 2 \\
\hline 20 & Ambroxol & 1 & 0.73 & 1.12 & 1.89 & 2.66 & 30 & $\mathrm{H}$ & $\mathrm{H}$ & 1 \\
\hline 21 & Amiloride & 3 & 1.01 & 2.16 & 2.12 & 0.11 & 5 & $\mathrm{H}$ & $\mathrm{L}$ & 3 \\
\hline 22 & Aminoglutethimide & 2 & 0.56 & 1.34 & 1.79 & 0.77 & 250 & $\mathrm{H}$ & $\mathrm{H}$ & 1 \\
\hline 23 & Aminophenazone & 1 & 0 & 1.79 & 1.88 & 1.04 & 300 & $\mathrm{H}$ & $\mathrm{H}$ & 1 \\
\hline 24 & Amisulpride & 4 & 0.5 & 2.18 & 3.16 & 1.8 & 200 & $\mathrm{~L}$ & $\mathrm{H}$ & 2 \\
\hline 25 & Amlodipine & 1 & 0.36 & 2.19 & 2.26 & 3.43 & 10 & $\mathrm{H}$ & $\mathrm{H}$ & 1 \\
\hline 26 & Amoxapine & 1 & 0.16 & 1.43 & 1.68 & 3.41 & 150 & $\mathrm{H}$ & $\mathrm{H}$ & 1 \\
\hline 27 & Amoxicillin & 3 & 1.55 & 2.9 & 3.59 & -1.87 & 500 & $\mathrm{H}$ & $\mathrm{L}$ & 3 \\
\hline 28 & Ampicillin & 3 & 1.06 & 2.62 & 3.01 & -1.2 & 500 & $\mathrm{H}$ & $\mathrm{L}$ & 3 \\
\hline 29 & Anastrozole & 1 & 0 & 1 & 2.38 & 1.29 & 1 & $\mathrm{H}$ & $\mathrm{H}$ & 1 \\
\hline 30 & Antipyrine; & 1 & 0 & 1.28 & 1.75 & -1.79 & 500 & $\mathrm{H}$ & $\mathrm{H}$ & 1 \\
\hline 31 & Aprepitant & 2 & 0.39 & 2.11 & 2.49 & 4.6 & 10 & $\mathrm{~L}$ & $\mathrm{H}$ & 2 \\
\hline 32 & Aripiprazole & 2 & 0.41 & 1.75 & 2.53 & 5.31 & 30 & $\mathrm{~L}$ & $\mathrm{H}$ & 2 \\
\hline 33 & Asenapine & 1 & 0 & 0.91 & 1.58 & 4.58 & 10 & $\mathrm{~L}$ & $\mathrm{H}$ & 2 \\
\hline
\end{tabular}


J Pharm Pharm Sci (www.cspsCanada.org) 22, 247 - 269, 2019

\begin{tabular}{|c|c|c|c|c|c|c|c|c|c|c|}
\hline 34 & Astemizole & 2 & 0.13 & 1.64 & 2.7 & 6.09 & 10 & $\mathrm{~L}$ & $\mathrm{H}$ & 2 \\
\hline 35 & Atenolol & 3 & 0.78 & 1.85 & 1.97 & -0.11 & 100 & $\mathrm{H}$ & $\mathrm{L}$ & 3 \\
\hline 36 & Atomoxetine & 1 & 0.13 & 0.9 & 1.36 & 3.94 & 60 & $\mathrm{H}$ & $\mathrm{H}$ & 1 \\
\hline 37 & Atovaquone & 4 & 0.31 & 1.21 & 2.54 & 6.35 & 250 & $\mathrm{~L}$ & $\mathrm{H}$ & 2 \\
\hline 38 & Atropine & 3 & 0.31 & 1.31 & 3.15 & 1.3 & 25 & $\mathrm{~L}$ & $\mathrm{H}$ & 2 \\
\hline 39 & Azathioprine & 1 & 0.35 & 1.56 & 2.86 & 0.51 & 100 & $\mathrm{~L}$ & $\mathrm{H}$ & 2 \\
\hline 40 & Azithromycin & 3 & 0.97 & 4.91 & 2.67 & 2.64 & 600 & $\mathrm{H}$ & $\mathrm{H}$ & 1 \\
\hline 41 & Bambuterol & 1 & 0.38 & 2.25 & 2.2 & 0.56 & 20 & $\mathrm{H}$ & $\mathrm{H}$ & 1 \\
\hline 42 & Benazepril & 1 & 0.71 & 2.08 & 2.75 & 1.82 & 40 & $\mathrm{H}$ & $\mathrm{H}$ & 1 \\
\hline 43 & Bendroflumethiazide & 3 & 1.01 & 1.84 & 2.89 & 1.73 & 10 & $\mathrm{~L}$ & $\mathrm{H}$ & 2 \\
\hline 44 & Benidipine & 1 & 0.13 & 2.13 & 2.99 & 7.41 & 8 & $\mathrm{H}$ & $\mathrm{H}$ & 1 \\
\hline 45 & Benserazide & 1 & 2.01 & 2.63 & 2.78 & -2.9 & 50 & $\mathrm{H}$ & $\mathrm{L}$ & 3 \\
\hline 46 & Benznidazole & 1 & 0.35 & 0.57 & 0.98 & 0.9 & 20 & $\mathrm{H}$ & $\mathrm{H}$ & 1 \\
\hline 47 & Bepridil & 1 & 0 & 1.32 & 1.81 & 6.2 & 400 & $\mathrm{~L}$ & $\mathrm{H}$ & 2 \\
\hline 48 & Beraprost & 1 & 1.2 & 1.51 & 2.03 & 2.04 & 0.04 & $\mathrm{H}$ & $\mathrm{H}$ & 1 \\
\hline 49 & Betamethasone & 1 & 0.8 & 1.97 & 2.95 & 1.79 & 0.75 & $\mathrm{H}$ & $\mathrm{H}$ & 1 \\
\hline 50 & Bexarotene & 2 & 0.57 & 0.67 & 1.29 & 8.19 & 75 & $\mathrm{~L}$ & $\mathrm{H}$ & 2 \\
\hline 51 & Bicalutamide & 2 & 0.71 & 1.63 & 3.05 & 2.71 & 50 & $\mathrm{~L}$ & $\mathrm{H}$ & 2 \\
\hline 52 & Biotin & 3 & 0.95 & 1.22 & 1.86 & -0.08 & 5 & $\mathrm{H}$ & $\mathrm{L}$ & 3 \\
\hline 53 & Biperiden & 1 & 0.31 & 1.17 & 1.32 & 4.94 & 2 & $\mathrm{H}$ & $\mathrm{H}$ & 1 \\
\hline 54 & Bopindolol & 1 & 0.46 & 1.48 & 2.14 & 4.98 & 2 & $\mathrm{H}$ & $\mathrm{H}$ & 1 \\
\hline 55 & Bosentan & 2 & 0.6 & 2.48 & 3.5 & 4.17 & 125 & $\mathrm{~L}$ & $\mathrm{H}$ & 2 \\
\hline 56 & Bromperidol & 1 & 0.31 & 1.45 & 2.16 & 4 & 10 & $\mathrm{~L}$ & $\mathrm{H}$ & 2 \\
\hline 57 & Budesonide & 1 & 0.48 & 2.16 & 3.23 & 2.91 & 3 & $\mathrm{H}$ & $\mathrm{H}$ & 1 \\
\hline 58 & Bumetanide & 3 & 1.16 & 1.7 & 1.92 & 3.37 & 500 & $\mathrm{H}$ & $\mathrm{H}$ & 1 \\
\hline 59 & Bupropion & 1 & 0.13 & 0.94 & 1.32 & 3.21 & 100 & $\mathrm{H}$ & $\mathrm{H}$ & 1 \\
\hline 60 & Buspirone & 2 & 0 & 2.16 & 2.18 & 2.19 & 10 & $\mathrm{H}$ & $\mathrm{H}$ & 1 \\
\hline 61 & Busulfan & 1 & 0 & 1.46 & 2.25 & -0.59 & 2 & $\mathrm{H}$ & $\mathrm{H}$ & 1 \\
\hline 62 & Butabarbital & 1 & 0.52 & 1.24 & 1.34 & 1.58 & 100 & $\mathrm{H}$ & $\mathrm{H}$ & 1 \\
\hline 63 & Butalbital & 1 & 0.52 & 1.3 & 1.4 & 1.63 & 50 & $\mathrm{H}$ & $\mathrm{H}$ & 1 \\
\hline 64 & Butorphanol & 1 & 0.73 & 1.32 & 1.42 & 3.73 & 5 & $\mathrm{H}$ & $\mathrm{H}$ & 1 \\
\hline 65 & Cadralazine & 3 & 0.57 & 1.84 & 2.14 & 0.93 & 10 & $\mathrm{H}$ & $\mathrm{H}$ & 1 \\
\hline 66 & Caffeine & 1 & 0 & 1.27 & 1.9 & -0.04 & 65 & $\mathrm{H}$ & $\mathrm{H}$ & 1 \\
\hline 67 & Candesartan cilexetil & 4 & 0.63 & 2.39 & 4.11 & 7.33 & 32 & $\mathrm{~L}$ & $\mathrm{H}$ & 2 \\
\hline 68 & Captopril & 3 & 0.57 & 1.13 & 1.77 & 0.89 & 100 & $\mathrm{H}$ & $\mathrm{H}$ & 1 \\
\hline 69 & Carbamazepine & 2 & 0.39 & 0.92 & 2.06 & 2.38 & 300 & $\mathrm{~L}$ & $\mathrm{H}$ & 2 \\
\hline 70 & Carbenicillin & 3 & 1.42 & 2.41 & 3.14 & 1.64 & 382 & $\mathrm{~L}$ & $\mathrm{~L}$ & 4 \\
\hline 71 & Carbidopa & 1 & 1.69 & 1.77 & 1.79 & -0.45 & 25 & $\mathrm{H}$ & $\mathrm{L}$ & 3 \\
\hline 72 & Cefaclor & 3 & 1.06 & 2.54 & 3.41 & -1.64 & 500 & $\mathrm{H}$ & $\mathrm{L}$ & 3 \\
\hline
\end{tabular}


J Pharm Pharm Sci (www.cspsCanada.org) 22, 247 - 269, 2019

\begin{tabular}{|c|c|c|c|c|c|c|c|c|c|c|}
\hline 73 & Cefadroxil & 3 & 1.55 & 2.82 & 3.48 & -2.51 & 1000 & $\mathrm{H}$ & $\mathrm{L}$ & 3 \\
\hline 74 & Cefamandole & 3 & 1.02 & 3.18 & 2.01 & 0.11 & 5 & $\mathrm{H}$ & $\mathrm{L}$ & 3 \\
\hline 75 & Cefdinir & 4 & 1.38 & 2.85 & 3.68 & -0.48 & 300 & $\mathrm{H}$ & $\mathrm{L}$ & 3 \\
\hline 76 & Cefditoren Pivoxil & 2 & 0.5 & 3.45 & 4.52 & 2.71 & 200 & $\mathrm{~L}$ & $\mathrm{H}$ & 2 \\
\hline 77 & Cefpodoxime & 4 & 1.07 & 2.95 & 3.67 & -0.41 & 200 & $\mathrm{H}$ & $\mathrm{L}$ & 3 \\
\hline 78 & Cefpodoxime Proxetil & 2 & 0.5 & 3.5 & 1.68 & 0.8 & 750 & $\mathrm{H}$ & $\mathrm{H}$ & 1 \\
\hline 79 & Cefprozil & 4 & 1.55 & 2.89 & 3.22 & -1.87 & 875 & $\mathrm{H}$ & $\mathrm{L}$ & 3 \\
\hline 80 & Ceftibuten & 4 & 1.64 & 2.77 & 4.02 & -1.21 & 400 & $\mathrm{~L}$ & $\mathrm{~L}$ & 4 \\
\hline 81 & Cefuroxime & 3 & 1.29 & 2.9 & 3.62 & 0.23 & 50 & $\mathrm{H}$ & $\mathrm{L}$ & 3 \\
\hline 82 & Cephradine & 3 & 1.06 & 2.59 & 1.06 & -1.73 & 250 & $\mathrm{H}$ & $\mathrm{L}$ & 3 \\
\hline 83 & Cerivastatin & 1 & 1.2 & 1.8 & 2.25 & 3.56 & 0.8 & $\mathrm{H}$ & $\mathrm{H}$ & 1 \\
\hline 84 & Cetirizine & 3 & 0.57 & 1.76 & 2.24 & 2.08 & 5 & $\mathrm{H}$ & $\mathrm{H}$ & 1 \\
\hline 85 & Chlorambucil & 1 & 0.57 & 0.8 & 1.6 & 3.63 & 2 & $\mathrm{H}$ & $\mathrm{H}$ & 1 \\
\hline 86 & Chlordiazepoxide & 1 & 0.31 & 1.41 & 1.31 & 3.79 & 25 & $\mathrm{H}$ & $\mathrm{H}$ & 1 \\
\hline 87 & Chlormethiazole & 1 & 0 & 0.3 & 0.91 & 1.68 & 192 & $\mathrm{H}$ & $\mathrm{H}$ & 1 \\
\hline 88 & Chloroquine & 3 & 0.13 & 1.29 & 1.63 & 5.06 & 500 & $\mathrm{~L}$ & $\mathrm{H}$ & 2 \\
\hline 89 & Chlorothiazide & 4 & 0.64 & 1.66 & 2.74 & -1 & 500 & $\mathrm{H}$ & $\mathrm{L}$ & 3 \\
\hline 90 & Chlorpheniramine & 1 & 0 & 1.02 & 1.49 & 3.15 & 4 & $\mathrm{H}$ & $\mathrm{H}$ & 1 \\
\hline 91 & Chlorpromazine & 1 & 0 & 0.99 & 1.45 & 5.3 & 4 & $\mathrm{H}$ & $\mathrm{H}$ & 1 \\
\hline 92 & Chlorthalidone & 4 & 1.01 & 1.98 & 3.05 & 0.45 & 100 & $\mathrm{~L}$ & $\mathrm{~L}$ & 4 \\
\hline 93 & Chlorzoxazone & 2 & 0.45 & 0.5 & 1.32 & 2.51 & 500 & $\mathrm{H}$ & $\mathrm{H}$ & 1 \\
\hline 94 & Cilazapril & 1 & 0.71 & 2.51 & 2.7 & 0.5 & 2.6 & $\mathrm{H}$ & $\mathrm{H}$ & 1 \\
\hline 95 & Cilazaprilat & 3 & 1.28 & 2.5 & 2.74 & 1.5 & 2.6 & $\mathrm{H}$ & $\mathrm{L}$ & 3 \\
\hline 96 & Cilostazol & 2 & 0.41 & 1.63 & 2.37 & 3.53 & 45 & $\mathrm{~L}$ & $\mathrm{H}$ & 2 \\
\hline 97 & Cimetidine & 3 & 0.74 & 1.86 & 1.87 & 0.19 & 800 & $\mathrm{H}$ & $\mathrm{L}$ & 3 \\
\hline 98 & Cinoxacin & 4 & 0.57 & 1.55 & 2.05 & 1.74 & 500 & $\mathrm{H}$ & $\mathrm{H}$ & 1 \\
\hline 99 & Cisapride & 2 & 0.5 & 2.17 & 3.15 & 3.81 & 20 & $\mathrm{~L}$ & $\mathrm{H}$ & 2 \\
\hline 100 & Citalopram & 2 & 0 & 1.08 & 2.25 & 3.13 & 20 & $\mathrm{~L}$ & $\mathrm{H}$ & 2 \\
\hline 101 & Clarithromycin & 3 & 0.8 & 4.49 & 2.97 & 2.37 & 500 & $\mathrm{H}$ & $\mathrm{H}$ & 1 \\
\hline 102 & Clavulanic Acid & 3 & 0.88 & 1.79 & 1.75 & -1.07 & 125 & $\mathrm{H}$ & $\mathrm{L}$ & 3 \\
\hline 103 & Clemastine & 1 & 0 & 0.97 & 1.55 & 5.45 & 2 & $\mathrm{H}$ & $\mathrm{H}$ & 1 \\
\hline 104 & Clodronic Acid & 4 & 1.25 & 2.22 & 1.49 & -0.14 & 800 & $\mathrm{H}$ & $\mathrm{L}$ & 3 \\
\hline 105 & Clofazimine & 2 & 0.19 & 1.28 & 2.34 & 7.7 & 50 & $\mathrm{~L}$ & $\mathrm{H}$ & 2 \\
\hline 106 & Clonazepam & 1 & 0.47 & 1.09 & 2.25 & 2.38 & 2 & $\mathrm{H}$ & $\mathrm{H}$ & 1 \\
\hline 107 & Cloxacillin & 4 & 0.84 & 2.32 & 3.27 & 2.52 & 250 & $\mathrm{~L}$ & $\mathrm{H}$ & 2 \\
\hline 108 & Clozapine & 2 & 0.2 & 1.65 & 1.66 & 3.71 & 100 & $\mathrm{H}$ & $\mathrm{H}$ & 1 \\
\hline 109 & Colchicine & 1 & 0.26 & 2.08 & 3.32 & 1.2 & 0.6 & $\mathrm{H}$ & $\mathrm{H}$ & 1 \\
\hline 110 & Cortisone & 1 & 0.41 & 1.9 & 1.63 & 1.3 & 0.4 & $\mathrm{H}$ & $\mathrm{H}$ & 1 \\
\hline 111 & Cyclizine & 1 & 0 & 1.21 & 1.55 & 3.8 & 50 & $\mathrm{H}$ & $\mathrm{H}$ & 1 \\
\hline
\end{tabular}


J Pharm Pharm Sci (www.cspsCanada.org) 22, 247 - 269, 2019

\begin{tabular}{|c|c|c|c|c|c|c|c|c|c|c|}
\hline 112 & Cycloserine & 3 & 0.48 & 1.46 & 1.42 & -1.19 & 250 & $\mathrm{H}$ & $\mathrm{L}$ & 3 \\
\hline 113 & Cyclosporine & 2 & 1.17 & 7.39 & 9.65 & 14.36 & 100 & $\mathrm{~L}$ & $\mathrm{H}$ & 2 \\
\hline 114 & Cyproheptadine & 1 & 0 & 0.83 & 1.85 & 5.3 & 10 & $\mathrm{~L}$ & $\mathrm{H}$ & 2 \\
\hline 115 & Dabigatran Etexilate & 1 & 0.34 & 3.07 & 4.1 & 4.13 & 110 & $\mathrm{~L}$ & $\mathrm{H}$ & 2 \\
\hline 116 & Dalfampridine & 3 & 0.23 & 0.71 & 1.21 & 0.32 & 10 & $\mathrm{H}$ & $\mathrm{H}$ & 1 \\
\hline 117 & Danazol & 2 & 0.4 & 1.03 & 2.58 & 3.93 & 5 & $\mathrm{H}$ & $\mathrm{H}$ & 1 \\
\hline 118 & Dantrolene & 1 & 0.24 & 1.44 & 2.5 & 1.63 & 100 & $\mathrm{~L}$ & $\mathrm{H}$ & 2 \\
\hline 119 & Darifenacin & 1 & 0.49 & 1.58 & 2.18 & 3.62 & 1.6 & $\mathrm{H}$ & $\mathrm{H}$ & 1 \\
\hline 120 & Darunavir & 2 & 0.64 & 2.86 & 3.74 & 2.89 & 600 & $\mathrm{~L}$ & $\mathrm{H}$ & 2 \\
\hline 121 & Dasatinib & 2 & 0.76 & 2.5 & 3.47 & 2.88 & 70 & $\mathrm{~L}$ & $\mathrm{H}$ & 2 \\
\hline 122 & Debrisoquine & 1 & 0.34 & 1.16 & 1.37 & 0.9 & 100 & $\mathrm{H}$ & $\mathrm{H}$ & 1 \\
\hline 123 & Delavirdine & 2 & 0.81 & 2.45 & 3.89 & 2.41 & 200 & $\mathrm{~L}$ & $\mathrm{H}$ & 2 \\
\hline 124 & Demeclocycline & 3 & 2.27 & 3.57 & 3.93 & -0.59 & 300 & $\mathrm{H}$ & $\mathrm{L}$ & 3 \\
\hline 125 & Desipramine & 1 & 0.13 & 0.9 & 1.58 & 4.47 & 200 & $\mathrm{~L}$ & $\mathrm{H}$ & 2 \\
\hline 126 & Desloratadine & 2 & 0.13 & 0.99 & 1.55 & 3.83 & 5 & $\mathrm{H}$ & $\mathrm{H}$ & 1 \\
\hline 127 & Desmethyldiazepam & 1 & 0.47 & 0.99 & 1 & 3.02 & 5 & $\mathrm{H}$ & $\mathrm{H}$ & 1 \\
\hline 128 & Desogestrel & 1 & 0.4 & 0.84 & 1.96 & 4.68 & 1.5 & $\mathrm{H}$ & $\mathrm{H}$ & 1 \\
\hline 129 & Dexamethasone & 1 & 0.8 & 1.97 & 2.95 & 1.79 & 5 & $\mathrm{H}$ & $\mathrm{H}$ & 1 \\
\hline 130 & Dexmethylphenidate & 1 & 0.13 & 0.94 & 1.29 & 2.56 & 10 & $\mathrm{H}$ & $\mathrm{H}$ & 1 \\
\hline 131 & Diazepam & 1 & 0 & 1.04 & 1.72 & 2.96 & 10 & $\mathrm{H}$ & $\mathrm{H}$ & 1 \\
\hline 132 & Diazoxide & 2 & 0.19 & 0.99 & 3.26 & 1.42 & 5 & $\mathrm{H}$ & $\mathrm{H}$ & 1 \\
\hline 133 & Diclofenac & 1 & 0.7 & 0.67 & 1.95 & 4.73 & 50 & $\mathrm{~L}$ & $\mathrm{H}$ & 2 \\
\hline 134 & Dicloxacillin & 3 & 0.84 & 2.26 & 1.56 & 2.98 & 750 & $\mathrm{H}$ & $\mathrm{H}$ & 1 \\
\hline 135 & Dicoumarol & 2 & 0.63 & 1.57 & 2.48 & 3.66 & 100 & $\mathrm{~L}$ & $\mathrm{H}$ & 2 \\
\hline 136 & Didanosine & 3 & 0.31 & 1.77 & 1.85 & -1.62 & 25 & $\mathrm{H}$ & $\mathrm{L}$ & 3 \\
\hline 137 & Diflunisal & 2 & 0.7 & 0.44 & 1.5 & 4.4 & 500 & $\mathrm{~L}$ & $\mathrm{H}$ & 2 \\
\hline 138 & Digitoxin & 3 & 1.27 & 4.02 & 4.2 & 2.85 & 0.1 & $\mathrm{H}$ & $\mathrm{H}$ & 1 \\
\hline 139 & Digoxin & 3 & 1.58 & 4.32 & 1.82 & 1.42 & 100 & $\mathrm{H}$ & $\mathrm{L}$ & 3 \\
\hline 140 & Dihydroquinidine & 1 & 0.23 & 1.76 & 1.52 & 3.27 & 40 & $\mathrm{H}$ & $\mathrm{H}$ & 1 \\
\hline 141 & Dilevalol & 1 & 1 & 1.72 & 2.3 & 2.5 & 50 & $\mathrm{H}$ & $\mathrm{H}$ & 1 \\
\hline 142 & Diloxanide furoate & 2 & 0.09 & 1.16 & 2.34 & 3.09 & 500 & $\mathrm{~L}$ & $\mathrm{H}$ & 2 \\
\hline 143 & Diltiazem & 1 & 0 & 2.22 & 2.14 & 3.65 & 120 & $\mathrm{H}$ & $\mathrm{H}$ & 1 \\
\hline 144 & Diphenhydramine & 1 & 0 & 0.95 & 1.43 & 3.45 & 50 & $\mathrm{H}$ & $\mathrm{H}$ & 1 \\
\hline 145 & Dipyridamole & 2 & 0.95 & 3.03 & 1.22 & 1.49 & 100 & $\mathrm{H}$ & $\mathrm{H}$ & 1 \\
\hline 146 & Disopyramide & 3 & 0.49 & 1.64 & 2.26 & 2.58 & 150 & $\mathrm{H}$ & $\mathrm{H}$ & 1 \\
\hline 147 & Disulfiram & 2 & 0 & 1.16 & 1.62 & 3.88 & 250 & $\mathrm{H}$ & $\mathrm{H}$ & 1 \\
\hline 148 & Dofetilide & 3 & 0.72 & 2.16 & 3.3 & 1.99 & 0.5 & $\mathrm{H}$ & $\mathrm{H}$ & 1 \\
\hline 149 & Dolasetron & 1 & 0.31 & 1.52 & 1.76 & 2.34 & 30 & $\mathrm{H}$ & $\mathrm{H}$ & 1 \\
\hline 150 & Donepezil & 2 & 0 & 1.5 & 2.17 & 4.6 & 125 & $\mathrm{H}$ & $\mathrm{H}$ & 1 \\
\hline
\end{tabular}


J Pharm Pharm Sci (www.cspsCanada.org) 22, 247 - 269, 2019

\begin{tabular}{|c|c|c|c|c|c|c|c|c|c|c|}
\hline 151 & Dosulepin & 1 & 0 & 0.89 & 1.46 & 4.53 & 75 & $\mathrm{~L}$ & $\mathrm{H}$ & 2 \\
\hline 152 & Doxazosin & 1 & 0.23 & 2.6 & 4.45 & 3.53 & 8 & $\mathrm{H}$ & $\mathrm{H}$ & 1 \\
\hline 153 & Doxepin & 1 & 0 & 0.98 & 1.46 & 4.09 & 100 & $\mathrm{H}$ & $\mathrm{H}$ & 1 \\
\hline 154 & Doxycycline & 3 & 2.1 & 3.47 & 3.88 & -0.51 & 40 & $\mathrm{H}$ & $\mathrm{L}$ & 3 \\
\hline 155 & Dronabinol & 2 & 0.5 & 0.71 & 1.04 & 7.24 & 10 & $\mathrm{~L}$ & $\mathrm{H}$ & 2 \\
\hline 156 & Dronedarone & 2 & 0.36 & 1.97 & 2.98 & 8.57 & 400 & $\mathrm{~L}$ & $\mathrm{H}$ & 2 \\
\hline 157 & Drospirenone & 2 & 0 & 1.24 & 3.29 & 2.84 & 3 & $\mathrm{H}$ & $\mathrm{H}$ & 1 \\
\hline 158 & Efavirenz & 2 & 0.42 & 0.61 & 1.13 & 4.67 & 50 & $\mathrm{H}$ & $\mathrm{H}$ & 1 \\
\hline 159 & Emtricitabine & 3 & 0.44 & 2 & 1.86 & -1.29 & 200 & $\mathrm{H}$ & $\mathrm{L}$ & 3 \\
\hline 160 & Enalapril & 1 & 0.71 & 1.92 & 2.61 & 0.67 & 20 & $\mathrm{H}$ & $\mathrm{H}$ & 1 \\
\hline 161 & Enalaprilat & 3 & 1.28 & 1.91 & 2.08 & 0.88 & 250 & $\mathrm{H}$ & $\mathrm{L}$ & 3 \\
\hline 162 & Enoxacin & 4 & 0.73 & 1.96 & 2.45 & -1.6 & 400 & $\mathrm{H}$ & $\mathrm{L}$ & 3 \\
\hline 163 & Entacapone & 2 & 0.58 & 1.38 & 2.85 & 1.76 & 200 & $\mathrm{~L}$ & $\mathrm{H}$ & 2 \\
\hline 164 & Eplerenone & 2 & 0 & 1.75 & 3.73 & 0.29 & 50 & $\mathrm{~L}$ & $\mathrm{H}$ & 2 \\
\hline 165 & Ergonovine & 1 & 0.81 & 1.89 & 2.47 & 1.23 & 0.2 & $\mathrm{H}$ & $\mathrm{H}$ & 1 \\
\hline 166 & Erythromycin & 3 & 1.05 & 4.63 & 3.04 & 1.61 & 500 & $\mathrm{H}$ & $\mathrm{L}$ & 3 \\
\hline 167 & Estazolam & 2 & 0 & 0.84 & 2.01 & 2.29 & 2 & $\mathrm{H}$ & $\mathrm{H}$ & 1 \\
\hline 168 & Estradiol & 1 & 0.81 & 0.95 & 2.3 & 3.78 & 2 & $\mathrm{H}$ & $\mathrm{H}$ & 1 \\
\hline 169 & Eszopiclone & 1 & 0 & 2.43 & 3.2 & 1.25 & 3 & $\mathrm{H}$ & $\mathrm{H}$ & 1 \\
\hline 170 & Ethambutol & 3 & 0.78 & 1.72 & 0.98 & 0.12 & 400 & $\mathrm{H}$ & $\mathrm{L}$ & 3 \\
\hline 171 & Ethchlorvynol & 2 & 0.4 & 0.47 & 0.82 & 1.57 & 750 & $\mathrm{H}$ & $\mathrm{H}$ & 1 \\
\hline 172 & Ethinylestradiol & 1 & 0.9 & 1.02 & 3.79 & 3.86 & 0.25 & $\mathrm{H}$ & $\mathrm{H}$ & 1 \\
\hline 173 & Ethosuximide & 1 & 0.34 & 0.93 & 0.94 & 0.4 & 250 & $\mathrm{H}$ & $\mathrm{H}$ & 1 \\
\hline 174 & Etidronic Acid & 3 & 1.56 & 2.54 & 1.55 & -2.54 & 400 & $\mathrm{H}$ & $\mathrm{L}$ & 3 \\
\hline 175 & Etodolac & 2 & 0.88 & 0.9 & 2.12 & 3.43 & 10 & $\mathrm{~L}$ & $\mathrm{H}$ & 2 \\
\hline 176 & Etoricoxib & 2 & 0 & 1.41 & 2.77 & 2.35 & 120 & $\mathrm{~L}$ & $\mathrm{H}$ & 2 \\
\hline 177 & Etravirine & 2 & 0.47 & 1.42 & 3.44 & 5.22 & 100 & $\mathrm{~L}$ & $\mathrm{H}$ & 2 \\
\hline 178 & Everolimus & 1 & 0.63 & 4.73 & 4.73 & 7.1 & 1 & $\mathrm{H}$ & $\mathrm{H}$ & 1 \\
\hline 179 & Exemestane & 2 & 0 & 1.14 & 2.6 & 3.28 & 25 & $\mathrm{~L}$ & $\mathrm{H}$ & 2 \\
\hline 180 & Famciclovir & 1 & 0.23 & 1.64 & 1.76 & 0.09 & 50 & $\mathrm{H}$ & $\mathrm{H}$ & 1 \\
\hline 181 & Felbamate & 4 & 0.89 & 1.19 & 2.12 & 0.5 & 600 & $\mathrm{H}$ & $\mathrm{L}$ & 3 \\
\hline 182 & Felodipine & 2 & 0.13 & 1.42 & 1.83 & 5.3 & 200 & $\mathrm{~L}$ & $\mathrm{H}$ & 2 \\
\hline 183 & Fenofibrate & 2 & 0 & 1.13 & 2.11 & 5.23 & 145 & $\mathrm{~L}$ & $\mathrm{H}$ & 2 \\
\hline 184 & Fentanyl & 1 & 0 & 1.33 & 2.82 & 3.62 & 15 & $\mathrm{~L}$ & $\mathrm{H}$ & 2 \\
\hline 185 & Fesoterodine & 1 & 0.31 & 1.58 & 1.75 & 4.36 & 8 & $\mathrm{H}$ & $\mathrm{H}$ & 1 \\
\hline 186 & Finasteride & 1 & 0.51 & 1.6 & 3.23 & 3.01 & 5 & $\mathrm{H}$ & $\mathrm{H}$ & 1 \\
\hline 187 & Flecainide & 3 & 0.41 & 1.32 & 1.68 & 3.66 & 150 & $\mathrm{H}$ & $\mathrm{H}$ & 1 \\
\hline 188 & Fleroxacin & 4 & 0.57 & 1.81 & 2.37 & -0.33 & 800 & $\mathrm{H}$ & $\mathrm{L}$ & 3 \\
\hline 189 & Fluconazole & 3 & 0.31 & 1.42 & 2.45 & -0.78 & 100 & $\mathrm{H}$ & $\mathrm{H}$ & 1 \\
\hline
\end{tabular}


J Pharm Pharm Sci (www.cspsCanada.org) 22, 247 - 269, 2019

\begin{tabular}{|c|c|c|c|c|c|c|c|c|c|c|}
\hline 190 & Flucytosine & 3 & 0.47 & 1.2 & 1.27 & -1.64 & 500 & $\mathrm{H}$ & $\mathrm{L}$ & 3 \\
\hline 191 & Flufenamic acid & 2 & 0.72 & 0.59 & 1.36 & 5.53 & 100 & $\mathrm{~L}$ & $\mathrm{H}$ & 2 \\
\hline 192 & Flunarizine & 2 & 0 & 1.37 & 2.06 & 6.34 & 10 & $\mathrm{~L}$ & $\mathrm{H}$ & 2 \\
\hline 193 & Flunitrazepam & 1 & 0 & 1.15 & 2.15 & 1.78 & 1 & $\mathrm{H}$ & $\mathrm{H}$ & 1 \\
\hline 194 & Fluoxetine & 1 & 0.13 & 0.78 & 1.19 & 4.57 & 20 & $\mathrm{H}$ & $\mathrm{H}$ & 1 \\
\hline 195 & Flurazepam & 1 & 0 & 1.55 & 1.89 & 4.22 & 30 & $\mathrm{H}$ & $\mathrm{H}$ & 1 \\
\hline 196 & Flurbiprofen & 2 & 0.57 & 0.58 & 1.51 & 3.75 & 100 & $\mathrm{~L}$ & $\mathrm{H}$ & 2 \\
\hline 197 & Flutamide & 2 & 0.53 & 0.68 & 1.76 & 3.34 & 125 & $\mathrm{~L}$ & $\mathrm{H}$ & 2 \\
\hline 198 & Fluvoxamine & 1 & 0.23 & 1.14 & 0.95 & 3.32 & 100 & $\mathrm{H}$ & $\mathrm{H}$ & 1 \\
\hline 199 & Folic Acid & 2 & 1.95 & 3.14 & 3.74 & -2.31 & 5 & $\mathrm{H}$ & $\mathrm{L}$ & 3 \\
\hline 200 & Fosfluconazole & 1 & 0.63 & 2.32 & 2.99 & -0.78 & 100 & $\mathrm{H}$ & $\mathrm{L}$ & 3 \\
\hline 201 & Fosinopril & 2 & 0.57 & 2.3 & 2.74 & 7.45 & 40 & $\mathrm{~L}$ & $\mathrm{H}$ & 2 \\
\hline 202 & Frovatriptan & 1 & 0.93 & 1.33 & 2.1 & 0.72 & 2.5 & $\mathrm{H}$ & $\mathrm{L}$ & 3 \\
\hline 203 & Furosemide & 4 & 1.25 & 1.5 & 2.37 & 1.9 & 80 & $\mathrm{~L}$ & $\mathrm{~L}$ & 4 \\
\hline 204 & Gabapentin & 3 & 0.78 & 0.93 & 0.99 & -0.66 & 800 & $\mathrm{H}$ & $\mathrm{L}$ & 3 \\
\hline 205 & Galantamine & 1 & 0.31 & 1.45 & 2.02 & 1.03 & 5 & $\mathrm{H}$ & $\mathrm{H}$ & 1 \\
\hline 206 & Gefitinib & 2 & 0.25 & 1.87 & 2.97 & 5.6 & 250 & $\mathrm{~L}$ & $\mathrm{H}$ & 2 \\
\hline 207 & Gemfibrozil & 2 & 0.57 & 0.71 & 1.07 & 3.94 & 600 & $\mathrm{H}$ & $\mathrm{H}$ & 1 \\
\hline 208 & Glibornuride & 1 & 0.84 & 1.64 & 2.46 & 3.7 & 25 & $\mathrm{~L}$ & $\mathrm{H}$ & 2 \\
\hline 209 & Gliclazide & 2 & 0.59 & 1.66 & 2.54 & 1.09 & 80 & $\mathrm{H}$ & $\mathrm{H}$ & 1 \\
\hline 210 & Glimepiride & 2 & 0.75 & 2.15 & 3.5 & 3.96 & 4 & $\mathrm{H}$ & $\mathrm{H}$ & 1 \\
\hline 211 & Glipizide & 2 & 0.85 & 2.19 & 1 & 2.57 & 250 & $\mathrm{H}$ & $\mathrm{H}$ & 1 \\
\hline 212 & Glyburide & 2 & 0.85 & 2.01 & 1.77 & 4.24 & 40 & $\mathrm{~L}$ & $\mathrm{H}$ & 2 \\
\hline 213 & Granisetron & 1 & 0.26 & 1.56 & 2.38 & 1.72 & 1 & $\mathrm{H}$ & $\mathrm{H}$ & 1 \\
\hline 214 & Griseofulvin & 2 & 0 & 1.58 & 1.87 & 1.91 & 100 & $\mathrm{H}$ & $\mathrm{H}$ & 1 \\
\hline 215 & Guanabenz & 1 & 0.48 & 1.2 & 1.02 & 2.98 & 16 & $\mathrm{H}$ & $\mathrm{H}$ & 1 \\
\hline 216 & Haloperidol & 2 & 0.31 & 1.45 & 2.08 & 3.85 & 20 & $\mathrm{~L}$ & $\mathrm{H}$ & 2 \\
\hline 217 & Hexobarbital & 1 & 0.24 & 1.33 & 1.5 & 1.63 & 250 & $\mathrm{H}$ & $\mathrm{H}$ & 1 \\
\hline 218 & Hydrochlorothiazide & 3 & 1.01 & 1.76 & 2.77 & -0.37 & 50 & $\mathrm{H}$ & $\mathrm{L}$ & 3 \\
\hline 219 & Hydrocodone & 1 & 0 & 1.42 & 2.12 & 1.13 & 10 & $\mathrm{H}$ & $\mathrm{H}$ & 1 \\
\hline 220 & Hydroflumethiazide & 3 & 1.01 & 1.72 & 2.44 & -0.21 & 50 & $\mathrm{H}$ & $\mathrm{L}$ & 3 \\
\hline 221 & Hydromorphone & 1 & 0.27 & 1.32 & 1.79 & 0.72 & 8 & $\mathrm{H}$ & $\mathrm{H}$ & 1 \\
\hline 222 & Hydroxychloroquine & 1 & 0.36 & 1.66 & 1.84 & 4.12 & 200 & $\mathrm{H}$ & $\mathrm{H}$ & 1 \\
\hline 223 & Hydroxyurea & 3 & 0.91 & 0.98 & 1.4 & -1.8 & 1000 & $\mathrm{H}$ & $\mathrm{L}$ & 3 \\
\hline 224 & Hydroxyzine & 1 & 0.23 & 1.8 & 2.41 & 4 & 30 & $\mathrm{~L}$ & $\mathrm{H}$ & 2 \\
\hline 225 & Hyoscyamine & 3 & 0.31 & 1.31 & 1.63 & 1.3 & 311 & $\mathrm{H}$ & $\mathrm{H}$ & 1 \\
\hline 226 & Ibandronate & 3 & 1.56 & 3.05 & 1.76 & -3.37 & 150 & $\mathrm{H}$ & $\mathrm{L}$ & 3 \\
\hline 227 & Ibuprofen & 2 & 0.57 & 0.51 & 1.01 & 3.68 & 800 & $\mathrm{H}$ & $\mathrm{H}$ & 1 \\
\hline 228 & Iloperidone & 2 & 0 & 1.73 & 2.85 & 4.27 & 12 & $\mathrm{~L}$ & $\mathrm{H}$ & 2 \\
\hline
\end{tabular}


J Pharm Pharm Sci (www.cspsCanada.org) 22, 247 - 269, 2019

\begin{tabular}{|c|c|c|c|c|c|c|c|c|c|c|}
\hline 229 & Imidapril & 1 & 0.71 & 2.22 & 2.85 & 1.53 & 10 & $\mathrm{H}$ & $\mathrm{H}$ & 1 \\
\hline 230 & Imipramine & 1 & 0 & 0.95 & 1.59 & 5.04 & 50 & $\mathrm{~L}$ & $\mathrm{H}$ & 2 \\
\hline 231 & Indobufen & 2 & 0.57 & 1.15 & 1.66 & 3.27 & 300 & $\mathrm{H}$ & $\mathrm{H}$ & 1 \\
\hline 232 & Indomethacin & 2 & 0.57 & 1.24 & 2.49 & 4.18 & 50 & $\mathrm{~L}$ & $\mathrm{H}$ & 2 \\
\hline 233 & Irbesartan & 2 & 0.63 & 1.69 & 2.71 & 6.04 & 300 & $\mathrm{~L}$ & $\mathrm{H}$ & 2 \\
\hline 234 & Isoniazid & 1 & 0.47 & 1.39 & 1.85 & -0.67 & 300 & $\mathrm{H}$ & $\mathrm{L}$ & 3 \\
\hline 235 & Isosorbide Dinitrate & 1 & 0 & 0.79 & 1.75 & 0.22 & 40 & $\mathrm{H}$ & $\mathrm{H}$ & 1 \\
\hline 236 & Isotretinoin & 2 & 0.57 & 0.8 & 0.98 & 6.74 & 10 & $\mathrm{~L}$ & $\mathrm{H}$ & 2 \\
\hline 237 & Itraconazole & 2 & 0 & 2.95 & 4.54 & 5.99 & 100 & $\mathrm{~L}$ & $\mathrm{H}$ & 2 \\
\hline 238 & Ivabradine & 1 & 0 & 2.35 & 3.25 & 3.97 & 7.5 & $\mathrm{H}$ & $\mathrm{H}$ & 1 \\
\hline 239 & Ketanserin & 2 & 0.26 & 1.82 & 2.01 & 3 & 25 & $\mathrm{~L}$ & $\mathrm{H}$ & 2 \\
\hline 240 & Ketoconazole & 2 & 0 & 2.22 & 3.76 & 3.64 & 200 & $\mathrm{~L}$ & $\mathrm{H}$ & 2 \\
\hline 241 & Ketorolac & 3 & 0.57 & 0.98 & 2.06 & 1.62 & 10 & $\mathrm{~L}$ & $\mathrm{H}$ & 2 \\
\hline 242 & Labetalol & 1 & 1 & 1.72 & 2.15 & 2.5 & 200 & $\mathrm{~L}$ & $\mathrm{H}$ & 2 \\
\hline 243 & Lacosamide & 3 & 0.53 & 1.48 & 2.55 & 0.39 & 50 & $\mathrm{H}$ & $\mathrm{H}$ & 1 \\
\hline 244 & Lamivudine & 3 & 0.44 & 2.02 & 1.92 & -1.46 & 300 & $\mathrm{H}$ & $\mathrm{L}$ & 3 \\
\hline 245 & Lamotrigine & 2 & 0.45 & 0.93 & 2.13 & 2.53 & 200 & $\mathrm{~L}$ & $\mathrm{H}$ & 2 \\
\hline 246 & Lansoprazole & 2 & 0.35 & 1.73 & 2.97 & 2.6 & 30 & $\mathrm{~L}$ & $\mathrm{H}$ & 2 \\
\hline 247 & Lapatinib ditosylate & 2 & 0.33 & 2.13 & 3.87 & 5.97 & 250 & $\mathrm{~L}$ & $\mathrm{H}$ & 2 \\
\hline 248 & Leflunomide & 2 & 0.47 & 0.81 & 2.17 & 2.32 & 10 & $\mathrm{~L}$ & $\mathrm{H}$ & 2 \\
\hline 249 & Lenalidomide & 4 & 0.56 & 1.91 & 2.53 & 0.53 & 200 & $\mathrm{H}$ & $\mathrm{H}$ & 1 \\
\hline 250 & Letrozole & 1 & 0 & 0.97 & 2.92 & 1.24 & 2.5 & $\mathrm{H}$ & $\mathrm{H}$ & 1 \\
\hline 251 & Leucovorin & 3 & 2.16 & 4.11 & 4.65 & -3.49 & 25 & $\mathrm{H}$ & $\mathrm{L}$ & 3 \\
\hline 252 & Levamisole & 1 & 0 & 0.88 & 1.01 & 1.84 & 50 & $\mathrm{H}$ & $\mathrm{H}$ & 1 \\
\hline 253 & Levetiracetam & 3 & 0.49 & 1.32 & 1.87 & -0.34 & 1000 & $\mathrm{H}$ & $\mathrm{L}$ & 3 \\
\hline 254 & Levocetirizine & 3 & 0.57 & 1.76 & 2.24 & 2.08 & 10 & $\mathrm{H}$ & $\mathrm{H}$ & 1 \\
\hline 255 & Levodopa & 1 & 1.56 & 1.44 & 1.77 & -2.82 & 250 & $\mathrm{H}$ & $\mathrm{L}$ & 3 \\
\hline 256 & Levonorgestrel & 4 & 0.4 & 1.07 & 2.45 & 3.31 & 0.75 & $\mathrm{H}$ & $\mathrm{H}$ & 1 \\
\hline 257 & Linezolid & 1 & 0.27 & 1.72 & 1.35 & 1.17 & 1.5 & $\mathrm{H}$ & $\mathrm{H}$ & 1 \\
\hline 258 & Loperamide & 3 & 0.31 & 1.88 & 2.9 & 4.66 & 2 & $\mathrm{H}$ & $\mathrm{H}$ & 1 \\
\hline 259 & Lopinavir & 2 & 0.92 & 2.89 & 4.57 & 6.1 & 200 & $\mathrm{~L}$ & $\mathrm{H}$ & 2 \\
\hline 260 & Loracarbef & 3 & 1.06 & 2.42 & 3.26 & -0.47 & 400 & $\mathrm{H}$ & $\mathrm{L}$ & 3 \\
\hline 261 & Loratadine & 2 & 0 & 1.14 & 2.17 & 5.05 & 10 & $\mathrm{~L}$ & $\mathrm{H}$ & 2 \\
\hline 262 & Lorazepam & 1 & 0.64 & 1.29 & 1.83 & 2.37 & 2 & $\mathrm{H}$ & $\mathrm{H}$ & 1 \\
\hline 263 & Lovastatin & 2 & 0.31 & 1.44 & 2.34 & 4.08 & 40 & $\mathrm{~L}$ & $\mathrm{H}$ & 2 \\
\hline 264 & Maprotiline & 1 & 0.13 & 0.68 & 1.27 & 4.52 & 75 & $\mathrm{H}$ & $\mathrm{H}$ & 1 \\
\hline 265 & Mebendazole & 2 & 0.71 & 1.38 & 2.76 & 3.08 & 100 & $\mathrm{~L}$ & $\mathrm{H}$ & 2 \\
\hline 266 & Mefenamic acid & 2 & 0.65 & 0.7 & 1.47 & 5.29 & 250 & $\mathrm{~L}$ & $\mathrm{H}$ & 2 \\
\hline 267 & Mefloquine & 2 & 0.38 & 1.22 & 1.04 & 3.67 & 250 & $\mathrm{H}$ & $\mathrm{H}$ & 1 \\
\hline
\end{tabular}


J Pharm Pharm Sci (www.cspsCanada.org) 22, 247 - 269, 2019

\begin{tabular}{|c|c|c|c|c|c|c|c|c|c|c|}
\hline 268 & Melatonin & 1 & 0.57 & 1.11 & 1.92 & 1.03 & 12 & $\mathrm{H}$ & $\mathrm{H}$ & 1 \\
\hline 269 & Meloxicam & 2 & 0.72 & 2.02 & 3.12 & 2.29 & 15 & $\mathrm{~L}$ & $\mathrm{H}$ & 2 \\
\hline 270 & Melphalan & 1 & 0.78 & 1.37 & 1.9 & -0.21 & 2 & $\mathrm{H}$ & $\mathrm{L}$ & 3 \\
\hline 271 & Meperidine & 1 & 0 & 0.97 & 3.13 & 2.23 & 20 & $\mathrm{H}$ & $\mathrm{H}$ & 1 \\
\hline 272 & Meprobamate & 1 & 0.89 & 1.12 & 1.62 & 0.92 & 400 & $\mathrm{H}$ & $\mathrm{L}$ & 3 \\
\hline 273 & Mercaptopurine & 2 & 0.43 & 0.7 & 1.31 & 0.82 & 50 & $\mathrm{H}$ & $\mathrm{H}$ & 1 \\
\hline 274 & Mesalamine & 2 & 0.93 & 0.7 & 1.52 & 1.06 & 1200 & $\mathrm{H}$ & $\mathrm{L}$ & 3 \\
\hline 275 & Metaxalone & 2 & 0.23 & 0.86 & 1.47 & 2.15 & 800 & $\mathrm{H}$ & $\mathrm{H}$ & 1 \\
\hline 276 & Metformin & 3 & 0.55 & 1.68 & 0.58 & -1.63 & 1000 & $\mathrm{H}$ & $\mathrm{L}$ & 3 \\
\hline 277 & Methazolamide & 3 & 0.44 & 2.01 & 2.5 & 0.09 & 500 & $\mathrm{H}$ & $\mathrm{H}$ & 1 \\
\hline 278 & Methotrexate & 3 & 1.85 & 2.82 & 4.23 & -0.53 & 15 & $\mathrm{~L}$ & $\mathrm{~L}$ & 4 \\
\hline 279 & Methyldopa & 3 & 1.56 & 1.45 & 1.73 & -2.26 & 500 & $\mathrm{H}$ & $\mathrm{L}$ & 3 \\
\hline 280 & Methylergonovine & 1 & 0.81 & 1.89 & 2.47 & 1.76 & 0.2 & $\mathrm{H}$ & $\mathrm{H}$ & 1 \\
\hline 281 & Methylphenidate & 1 & 0.13 & 0.94 & 1.29 & 2.56 & 20 & $\mathrm{H}$ & $\mathrm{H}$ & 1 \\
\hline 282 & Methylprednisolone & 1 & 0.73 & 2 & 3 & 1.74 & 32 & $\mathrm{~L}$ & $\mathrm{H}$ & 2 \\
\hline 283 & Metoprolol & 1 & 0.29 & 1.52 & 2.9 & 1.49 & 75 & $\mathrm{H}$ & $\mathrm{H}$ & 1 \\
\hline 284 & Metronidazole & 1 & 0.31 & 0.86 & 1.75 & -0.46 & 500 & $\mathrm{H}$ & $\mathrm{H}$ & 1 \\
\hline 285 & Mexiletine & 1 & 0.23 & 0.9 & 3.71 & 2.57 & 10 & $\mathrm{~L}$ & $\mathrm{H}$ & 2 \\
\hline 286 & Mianserin & 1 & 0 & 1.03 & 1.35 & 3.76 & 50 & $\mathrm{H}$ & $\mathrm{H}$ & 1 \\
\hline 287 & Miglitol & 3 & 1.18 & 2.3 & 1.57 & -1.26 & 100 & $\mathrm{H}$ & $\mathrm{L}$ & 3 \\
\hline 288 & Miglustat & 3 & 0.93 & 1.95 & 1.36 & 0.91 & 100 & $\mathrm{H}$ & $\mathrm{L}$ & 3 \\
\hline 289 & Milnacipran & 3 & 0.21 & 1.33 & 2.32 & 1.91 & 500 & $\mathrm{H}$ & $\mathrm{H}$ & 1 \\
\hline 290 & Minoxidil & 1 & 0.52 & 2.07 & 1.05 & -0.72 & 10 & $\mathrm{H}$ & $\mathrm{L}$ & 3 \\
\hline 291 & Mirtazapine & 1 & 0 & 1.52 & 2.16 & 2.81 & 45 & $\mathrm{H}$ & $\mathrm{H}$ & 1 \\
\hline 292 & Misoprostol & 1 & 0.63 & 1.43 & 1.77 & 3.07 & 0.2 & $\mathrm{H}$ & $\mathrm{H}$ & 1 \\
\hline 293 & Mizolastine & 2 & 0.39 & 2.15 & 3.09 & 2.84 & 10 & $\mathrm{~L}$ & $\mathrm{H}$ & 2 \\
\hline 294 & Modafinil & 2 & 0.49 & 1.47 & 3.2 & 0.94 & 200 & $\mathrm{~L}$ & $\mathrm{H}$ & 2 \\
\hline 295 & Molindone & 1 & 0.31 & 1.29 & 1.57 & 2.57 & 50 & $\mathrm{H}$ & $\mathrm{H}$ & 1 \\
\hline 296 & Mycophenolate mofetil & 2 & 0.13 & 1.66 & 1.96 & 2.98 & 500 & $\mathrm{H}$ & $\mathrm{H}$ & 1 \\
\hline 297 & Nabumetone & 2 & 0 & 0.7 & 3.34 & 2.98 & 500 & $\mathrm{~L}$ & $\mathrm{H}$ & 2 \\
\hline 298 & Nadolol & 3 & 0.83 & 1.9 & 1.56 & 0.38 & 160 & $\mathrm{H}$ & $\mathrm{L}$ & 3 \\
\hline 299 & Nalidixic acid & 2 & 0.57 & 1.34 & 1.1 & 1.02 & 1200 & $\mathrm{H}$ & $\mathrm{H}$ & 1 \\
\hline 300 & Naproxen & 2 & 0.57 & 0.75 & 1.49 & 2.82 & 500 & $\mathrm{H}$ & $\mathrm{H}$ & 1 \\
\hline 301 & Naratriptan & 3 & 0.68 & 1.62 & 2.14 & 1.7 & 2.5 & $\mathrm{H}$ & $\mathrm{H}$ & 1 \\
\hline 302 & Nateglinide & 2 & 0.83 & 1.12 & 2.02 & 4.3 & 120 & $\mathrm{~L}$ & $\mathrm{H}$ & 2 \\
\hline 303 & Nefazodone & 2 & 0 & 2.12 & 2.8 & 5.73 & 100 & $\mathrm{~L}$ & $\mathrm{H}$ & 2 \\
\hline 304 & Nefopam & 1 & 0 & 0.92 & 1.46 & 2.91 & 30 & $\mathrm{H}$ & $\mathrm{H}$ & 1 \\
\hline 305 & Nelfinavir & 2 & 1.27 & 2.81 & 3.72 & 5.84 & 200 & $\mathrm{~L}$ & $\mathrm{H}$ & 2 \\
\hline 306 & Neostigmine & 3 & 0 & 0.73 & 1.21 & -2.81 & 15 & $\mathrm{H}$ & $\mathrm{L}$ & 3 \\
\hline
\end{tabular}


J Pharm Pharm Sci (www.cspsCanada.org) 22, 247 - 269, 2019

\begin{tabular}{|c|c|c|c|c|c|c|c|c|c|c|}
\hline 307 & Nevirapine & 2 & 0.42 & 1.37 & 3.74 & 2.65 & 100 & $\mathrm{~L}$ & $\mathrm{H}$ & 2 \\
\hline 308 & Niacin & 1 & 0.57 & 0.73 & 4.08 & 0.8 & 200 & $\mathrm{~L}$ & $\mathrm{H}$ & 2 \\
\hline 309 & Niacinamide & 1 & 0.49 & 0.94 & 3.16 & 0.8 & 300 & $\mathrm{H}$ & $\mathrm{H}$ & 1 \\
\hline 310 & Nicardipine & 1 & 0.13 & 2.12 & 2.91 & 5.23 & 30 & $\mathrm{~L}$ & $\mathrm{H}$ & 2 \\
\hline 311 & Niclosamide & 4 & 0.77 & 0.78 & 2.67 & 4.34 & 500 & $\mathrm{~L}$ & $\mathrm{H}$ & 2 \\
\hline 312 & Nicorandil & 1 & 0.27 & 1.09 & 2.22 & 0.75 & 20 & $\mathrm{H}$ & $\mathrm{H}$ & 1 \\
\hline 313 & Nicotine & 1 & 0 & 0.91 & 1.03 & 0.88 & 4 & $\mathrm{H}$ & $\mathrm{H}$ & 1 \\
\hline 314 & Nifedipine & 2 & 0.13 & 1.53 & 1.87 & 3.13 & 40 & $\mathrm{~L}$ & $\mathrm{H}$ & 2 \\
\hline 315 & Nilotinib & 2 & 0.61 & 2.06 & 3.62 & 5.84 & 625 & $\mathrm{~L}$ & $\mathrm{H}$ & 2 \\
\hline 316 & Nilvadipine & 2 & 0.13 & 1.74 & 2.79 & 3.04 & 2 & $\mathrm{H}$ & $\mathrm{H}$ & 1 \\
\hline 317 & Nimesulide & 2 & 0.43 & 1.1 & 2.68 & 3.21 & 100 & $\mathrm{~L}$ & $\mathrm{H}$ & 2 \\
\hline 318 & Nimodipine & 2 & 0.13 & 1.79 & 2.01 & 4 & 100 & $\mathrm{H}$ & $\mathrm{H}$ & 1 \\
\hline 319 & Nitrazepam & 2 & 0.47 & 1.1 & 1.67 & 2.32 & 100 & $\mathrm{H}$ & $\mathrm{H}$ & 1 \\
\hline 320 & Nitrendipine & 2 & 0.13 & 1.54 & 2.26 & 3.73 & 20 & $\mathrm{~L}$ & $\mathrm{H}$ & 2 \\
\hline 321 & Nizatidine & 3 & 0.27 & 2.11 & 2.05 & -0.16 & 300 & $\mathrm{H}$ & $\mathrm{H}$ & 1 \\
\hline 322 & Norelgestromin & 2 & 0.71 & 1.2 & 2.1 & 4.1 & 6 & $\mathrm{H}$ & $\mathrm{H}$ & 1 \\
\hline 323 & Norethindrone & 1 & 0.4 & 1.07 & 2.44 & 2.78 & 0.35 & $\mathrm{H}$ & $\mathrm{H}$ & 1 \\
\hline 324 & Norethindrone acetate & 2 & 0.09 & 1.13 & 1.81 & 3.93 & 30 & $\mathrm{~L}$ & $\mathrm{H}$ & 2 \\
\hline 325 & Norfloxacin & 4 & 0.73 & 1.84 & 2.43 & -0.78 & 400 & $\mathrm{H}$ & $\mathrm{L}$ & 3 \\
\hline 326 & Norgestimate & 1 & 0.4 & 1.25 & 2.23 & 5.06 & 0.25 & $\mathrm{H}$ & $\mathrm{H}$ & 1 \\
\hline 327 & Norgestrel & 1 & 0.4 & 1.07 & 2.45 & 3.31 & 0.5 & $\mathrm{H}$ & $\mathrm{H}$ & 1 \\
\hline 328 & Nortriptyline & 1 & 0.13 & 0.72 & 1.3 & 4.32 & 75 & $\mathrm{H}$ & $\mathrm{H}$ & 1 \\
\hline 329 & Nystatin & 3 & 3.55 & 5.93 & 5.02 & -3.2 & 200 & $\mathrm{H}$ & $\mathrm{L}$ & 3 \\
\hline 330 & Ofloxacin & 3 & 0.57 & 2.05 & 2.58 & -0.51 & 400 & $\mathrm{H}$ & $\mathrm{L}$ & 3 \\
\hline 331 & Olanzapine & 2 & 0.13 & 1.79 & 1.55 & 3.01 & 40 & $\mathrm{H}$ & $\mathrm{H}$ & 1 \\
\hline 332 & Olmesartan medoxomil & 1 & 0.95 & 2.61 & 3.72 & 2.91 & 40 & $\mathrm{~L}$ & $\mathrm{H}$ & 2 \\
\hline 333 & Ondansetron & 1 & 0 & 1.08 & 2.22 & 2.72 & 8 & $\mathrm{H}$ & $\mathrm{H}$ & 1 \\
\hline 334 & Orlistat & 4 & 0.26 & 1.54 & 2.75 & 8.61 & 120 & $\mathrm{~L}$ & $\mathrm{H}$ & 2 \\
\hline 335 & Orphenadrine & 1 & 0 & 0.95 & 1.38 & 3.9 & 100 & $\mathrm{H}$ & $\mathrm{H}$ & 1 \\
\hline 336 & Oxaprozin & 2 & 0.57 & 0.92 & 2.17 & 2.95 & 600 & $\mathrm{~L}$ & $\mathrm{H}$ & 2 \\
\hline 337 & Oxazepam & 2 & 0.38 & 1.49 & 2.33 & 2.31 & 30 & $\mathrm{~L}$ & $\mathrm{H}$ & 2 \\
\hline 338 & Oxcarbazepine & 2 & 0.39 & 1.16 & 2.45 & 1.21 & 600 & $\mathrm{~L}$ & $\mathrm{H}$ & 2 \\
\hline 339 & Oxprenolol & 1 & 0.29 & 1.61 & 1.65 & 2.09 & 6 & $\mathrm{H}$ & $\mathrm{H}$ & 1 \\
\hline 340 & Oxycodone & 1 & 0.23 & 1.8 & 2.28 & -0.04 & 80 & $\mathrm{H}$ & $\mathrm{H}$ & 1 \\
\hline 341 & Oxymorphone & 1 & 0.5 & 1.69 & 1.95 & -0.48 & 40 & $\mathrm{H}$ & $\mathrm{L}$ & 3 \\
\hline 342 & Paliperidone & 4 & 0.31 & 2.01 & 2.48 & 1.12 & 9 & $\mathrm{H}$ & $\mathrm{H}$ & 1 \\
\hline 343 & Paroxetine & 1 & 0.13 & 1.23 & 3.84 & 4.24 & 6 & $\mathrm{H}$ & $\mathrm{H}$ & 1 \\
\hline 344 & Pefloxacin & 1 & 0.57 & 1.91 & 2.42 & -0.32 & 400 & $\mathrm{H}$ & $\mathrm{L}$ & 3 \\
\hline 345 & Penicillamine & 3 & 0.78 & 1.09 & 3.06 & -1.73 & 500 & $\mathrm{H}$ & $\mathrm{L}$ & 3 \\
\hline
\end{tabular}


J Pharm Pharm Sci (www.cspsCanada.org) 22, 247 - 269, 2019

\begin{tabular}{|c|c|c|c|c|c|c|c|c|c|c|}
\hline 346 & Penicillin V & 4 & 0.84 & 2.27 & 2.86 & 1.94 & 500 & $\mathrm{H}$ & $\mathrm{H}$ & 1 \\
\hline 347 & Pentazocine & 2 & 0.5 & 1.04 & 1.38 & 4.67 & 600 & $\mathrm{~L}$ & $\mathrm{H}$ & 2 \\
\hline 348 & Pentoxifylline & 1 & 0 & 1.59 & 2.42 & 0.12 & 400 & $\mathrm{H}$ & $\mathrm{H}$ & 1 \\
\hline 349 & Perhexiline & 2 & 0.13 & 0.56 & 0.62 & 7.15 & 100 & $\mathrm{H}$ & $\mathrm{H}$ & 1 \\
\hline 350 & Perindopril erbumine & 1 & 0.71 & 1.88 & 2.23 & 1.21 & 8 & $\mathrm{H}$ & $\mathrm{H}$ & 1 \\
\hline 351 & Phenacetin & 2 & 0.41 & 0.87 & 1.55 & 1.71 & 500 & $\mathrm{H}$ & $\mathrm{H}$ & 1 \\
\hline 352 & Phenobarbital & 1 & 0.52 & 1.29 & 1.81 & 1.37 & 60 & $\mathrm{H}$ & $\mathrm{H}$ & 1 \\
\hline 353 & Phenylbutazone & 1 & 0 & 1.63 & 2.45 & 3.39 & 100 & $\mathrm{~L}$ & $\mathrm{H}$ & 2 \\
\hline 354 & Phenylethylmalonamide & 3 & 0.97 & 1.25 & 2.41 & 0.01 & 250 & $\mathrm{H}$ & $\mathrm{L}$ & 3 \\
\hline 355 & Pimozide & 1 & 0.33 & 1.44 & 2.6 & 6.4 & 2 & $\mathrm{H}$ & $\mathrm{H}$ & 1 \\
\hline 356 & Pindolol & 3 & 0.6 & 1.51 & 1.38 & 1.67 & 50 & $\mathrm{H}$ & $\mathrm{H}$ & 1 \\
\hline 357 & Pioglitazone & 2 & 0.34 & 1.64 & 2.44 & 3.53 & 100 & $\mathrm{~L}$ & $\mathrm{H}$ & 2 \\
\hline 358 & Piperazine & 3 & 0.29 & 0.89 & 0.63 & -1.48 & 500 & $\mathrm{H}$ & $\mathrm{L}$ & 3 \\
\hline 359 & Piracetam & 3 & 0.49 & 1.28 & 1.88 & -1.18 & 800 & $\mathrm{H}$ & $\mathrm{L}$ & 3 \\
\hline 360 & Pirenzepine & 3 & 0.42 & 2.42 & 3.13 & -0.35 & 50 & $\mathrm{H}$ & $\mathrm{H}$ & 1 \\
\hline 361 & Piroxicam & 2 & 0.72 & 2.12 & 3.12 & 1.89 & 20 & $\mathrm{~L}$ & $\mathrm{H}$ & 2 \\
\hline 362 & Pramipexole & 3 & 0.36 & 0.97 & 2.69 & 1.17 & 600 & $\mathrm{~L}$ & $\mathrm{H}$ & 2 \\
\hline 363 & Prasugrel & 2 & 0 & 1.3 & 1.57 & 3.43 & 600 & $\mathrm{H}$ & $\mathrm{H}$ & 1 \\
\hline 364 & Prazepam & 2 & 0 & 1.05 & 2.38 & 3.93 & 200 & $\mathrm{~L}$ & $\mathrm{H}$ & 2 \\
\hline 365 & Prazosin & 1 & 0.23 & 2.17 & 3.59 & 2.03 & 5 & $\mathrm{H}$ & $\mathrm{H}$ & 1 \\
\hline 366 & Prednisolone & 1 & 0.72 & 2 & 4.46 & 1.42 & 0.25 & $\mathrm{H}$ & $\mathrm{H}$ & 1 \\
\hline 367 & Prednisone & 2 & 0.41 & 1.97 & 3.25 & 1.66 & 50 & $\mathrm{~L}$ & $\mathrm{H}$ & 2 \\
\hline 368 & Primaquine & 1 & 0.34 & 1.49 & 1.76 & 2.6 & 15 & $\mathrm{H}$ & $\mathrm{H}$ & 1 \\
\hline 369 & Primidone & 2 & 0.51 & 1.45 & 2.65 & 0.88 & 8 & $\mathrm{H}$ & $\mathrm{H}$ & 1 \\
\hline 370 & Probenecid & 2 & 0.57 & 1.29 & 2.65 & 3.37 & 2 & $\mathrm{H}$ & $\mathrm{H}$ & 1 \\
\hline 371 & Probucol & 2 & 0.62 & 1.2 & 1.38 & 10.97 & 500 & $\mathrm{~L}$ & $\mathrm{H}$ & 2 \\
\hline 372 & Procainamide & 3 & 0.5 & 1.49 & 2.11 & 1.42 & 1000 & $\mathrm{H}$ & $\mathrm{H}$ & 1 \\
\hline 373 & Prochlorperazine & 1 & 0 & 1.47 & 2.11 & 4.38 & 10 & $\mathrm{~L}$ & $\mathrm{H}$ & 2 \\
\hline 374 & Progesterone & 2 & 0 & 1.04 & 2.49 & 3.78 & 200 & $\mathrm{~L}$ & $\mathrm{H}$ & 2 \\
\hline 375 & Proguanil & 1 & 0.74 & 1.59 & 1.19 & 2.53 & 100 & $\mathrm{H}$ & $\mathrm{H}$ & 1 \\
\hline 376 & Promazine & 1 & 0 & 1.06 & 1.72 & 4.4 & 50 & $\mathrm{~L}$ & $\mathrm{H}$ & 2 \\
\hline 377 & Promethazine & 1 & 0 & 1.09 & 1.74 & 4.4 & 100 & $\mathrm{~L}$ & $\mathrm{H}$ & 2 \\
\hline 378 & Propylthiouracil & 1 & 0.39 & 1.03 & 1.74 & 0.97 & 50 & $\mathrm{H}$ & $\mathrm{H}$ & 1 \\
\hline 379 & Protriptyline & 1 & 0.13 & 0.73 & 1.34 & 4.87 & 10 & $\mathrm{~L}$ & $\mathrm{H}$ & 2 \\
\hline 380 & Pseudoephedrine & 3 & 0.38 & 1.12 & 0.94 & 0.89 & 120 & $\mathrm{H}$ & $\mathrm{H}$ & 1 \\
\hline 381 & Pyrazinamide & 1 & 0.49 & 1.04 & 1.68 & -0.68 & 500 & $\mathrm{H}$ & $\mathrm{L}$ & 3 \\
\hline 382 & Pyridostigmine & 3 & 0 & 0.7 & 1.23 & -4.26 & 60 & $\mathrm{H}$ & $\mathrm{L}$ & 3 \\
\hline 383 & Pyrimethamine & 3 & 0.45 & 0.99 & 2.69 & 3 & 40 & $\mathrm{~L}$ & $\mathrm{H}$ & 2 \\
\hline 384 & Quinacrine & 1 & 0.13 & 1.56 & 2.05 & 6.72 & 100 & $\mathrm{~L}$ & $\mathrm{H}$ & 2 \\
\hline
\end{tabular}


J Pharm Pharm Sci (www.cspsCanada.org) 22, 247 - 269, 2019

\begin{tabular}{|c|c|c|c|c|c|c|c|c|c|c|}
\hline 385 & Quinapril & 2 & 0.71 & 2 & 3.01 & 1.74 & 40 & $\mathrm{~L}$ & $\mathrm{H}$ & 2 \\
\hline 386 & Raloxifene & 2 & 1 & 1.85 & 3.12 & 6.86 & 60 & $\mathrm{~L}$ & $\mathrm{H}$ & 2 \\
\hline 387 & Ramelteon & 1 & 0.26 & 0.89 & 1.67 & 2.49 & 8 & $\mathrm{H}$ & $\mathrm{H}$ & 1 \\
\hline 388 & Ranitidine & 3 & 0.27 & 1.97 & 1.74 & 0.67 & 300 & $\mathrm{H}$ & $\mathrm{H}$ & 1 \\
\hline 389 & Ranolazine & 2 & 0.4 & 2.69 & 3.04 & 1.01 & 1000 & $\mathrm{H}$ & $\mathrm{H}$ & 1 \\
\hline 390 & Reboxetine & 1 & 0.16 & 1.36 & 2.19 & 3.26 & 6 & $\mathrm{H}$ & $\mathrm{H}$ & 1 \\
\hline 391 & Reserpine & 1 & 0.31 & 2.76 & 2.43 & 3.86 & 1 & $\mathrm{H}$ & $\mathrm{H}$ & 1 \\
\hline 392 & Ribavirin & 1 & 1.23 & 2.28 & 2.83 & -2.85 & 200 & $\mathrm{H}$ & $\mathrm{L}$ & 3 \\
\hline 393 & Rifabutin & 2 & 1.31 & 4.39 & 4.43 & 4.73 & 150 & $\mathrm{~L}$ & $\mathrm{H}$ & 2 \\
\hline 394 & Rifampin & 2 & 2.05 & 5.36 & 5.22 & 3.71 & 300 & $\mathrm{~L}$ & $\mathrm{H}$ & 2 \\
\hline 395 & Rifaximin & 4 & 1.99 & 3.89 & 4.88 & 7.24 & 550 & $\mathrm{~L}$ & $\mathrm{H}$ & 2 \\
\hline 396 & Riluzole & 1 & 0.23 & 0.67 & 1.45 & 3.24 & 50 & $\mathrm{H}$ & $\mathrm{H}$ & 1 \\
\hline 397 & Risedronate & 3 & 1.56 & 2.91 & 2.15 & -2.62 & 150 & $\mathrm{H}$ & $\mathrm{L}$ & 3 \\
\hline 398 & Risperidone & 1 & 0 & 1.7 & 2.23 & 2.71 & 4 & $\mathrm{H}$ & $\mathrm{H}$ & 1 \\
\hline 399 & Rivastigmine & 1 & 0 & 1.23 & 2.69 & 2.1 & 4 & $\mathrm{H}$ & $\mathrm{H}$ & 1 \\
\hline 400 & Rizatriptan & 1 & 0.31 & 1.28 & 2.05 & 0.99 & 10 & $\mathrm{H}$ & $\mathrm{H}$ & 1 \\
\hline 401 & Rofecoxib & 2 & 0 & 1.15 & 2.43 & 1.8 & 50 & $\mathrm{~L}$ & $\mathrm{H}$ & 2 \\
\hline 402 & Ropinirole & 1 & 0.41 & 1.27 & 1.41 & 2.8 & 5 & $\mathrm{H}$ & $\mathrm{H}$ & 1 \\
\hline 403 & Roxithromycin & 4 & 1.05 & 5.12 & 2.9 & 2.29 & 300 & $\mathrm{H}$ & $\mathrm{H}$ & 1 \\
\hline 404 & Salicylic Acid & 1 & 0.7 & 0.4 & 1.94 & 1.02 & 1000 & $\mathrm{H}$ & $\mathrm{H}$ & 1 \\
\hline 405 & Saxagliptin & 3 & 0.45 & 0.99 & 4.03 & 0.11 & 400 & $\mathrm{~L}$ & $\mathrm{H}$ & 2 \\
\hline 406 & Scopolamine & 1 & 0.31 & 1.55 & 1.84 & 0.29 & 10 & $\mathrm{H}$ & $\mathrm{H}$ & 1 \\
\hline 407 & Secobarbital & 1 & 0.52 & 1.3 & 2.84 & 2.16 & 12 & $\mathrm{~L}$ & $\mathrm{H}$ & 2 \\
\hline 408 & Selegiline & 1 & 0.09 & 0.71 & 1.71 & 3.02 & 10 & $\mathrm{H}$ & $\mathrm{H}$ & 1 \\
\hline 409 & Sibutramine & 1 & 0 & 0.7 & 0.92 & 5.59 & 15 & $\mathrm{H}$ & $\mathrm{H}$ & 1 \\
\hline 410 & Sildenafil & 1 & 0.08 & 2.67 & 2.94 & 1.98 & 100 & $\mathrm{H}$ & $\mathrm{H}$ & 1 \\
\hline 411 & Simvastatin & 2 & 0.31 & 1.45 & 2.29 & 4.48 & 80 & $\mathrm{~L}$ & $\mathrm{H}$ & 2 \\
\hline 412 & Sitafloxacin & 3 & 0.8 & 1.93 & 2.71 & -1.25 & 50 & $\mathrm{H}$ & $\mathrm{L}$ & 3 \\
\hline 413 & Sitagliptin & 3 & 0.21 & 1.61 & 2.52 & 0.69 & 100 & $\mathrm{H}$ & $\mathrm{H}$ & 1 \\
\hline 414 & Sorafenib tosylate & 2 & 0.97 & 1.81 & 3.36 & 5.46 & 200 & $\mathrm{~L}$ & $\mathrm{H}$ & 2 \\
\hline 415 & Sotalol & 3 & 0.74 & 1.74 & 1.98 & 0.23 & 240 & $\mathrm{H}$ & $\mathrm{L}$ & 3 \\
\hline 416 & Sparfloxacin & 1 & 0.97 & 2.08 & 2.64 & -0.61 & 200 & $\mathrm{H}$ & $\mathrm{L}$ & 3 \\
\hline 417 & Spironolactone & 2 & 0 & 1.82 & 2.29 & 2.65 & 200 & $\mathrm{~L}$ & $\mathrm{H}$ & 2 \\
\hline 418 & Stavudine & 3 & 0.47 & 1.65 & 1.96 & -0.49 & 40 & $\mathrm{H}$ & $\mathrm{L}$ & 3 \\
\hline 419 & Sulfamethizole & 4 & 0.59 & 1.26 & 2.71 & 0.42 & 500 & $\mathrm{~L}$ & $\mathrm{H}$ & 2 \\
\hline 420 & Sulfamethoxazole & 2 & 0.59 & 1.21 & 2.43 & 0.56 & 800 & $\mathrm{~L}$ & $\mathrm{H}$ & 2 \\
\hline 421 & Sulfasalazine & 2 & 1.06 & 2.21 & 3.42 & 3.88 & 500 & $\mathrm{~L}$ & $\mathrm{H}$ & 2 \\
\hline 422 & Sulfinpyrazone & 2 & 0 & 2.39 & 4.1 & 1.66 & 200 & $\mathrm{~L}$ & $\mathrm{H}$ & 2 \\
\hline 423 & Sulfisoxazole & 4 & 0.59 & 1.31 & 2.44 & 0.22 & 500 & $\mathrm{H}$ & $\mathrm{H}$ & 1 \\
\hline
\end{tabular}


J Pharm Pharm Sci (www.cspsCanada.org) 22, 247 - 269, 2019

\begin{tabular}{|c|c|c|c|c|c|c|c|c|c|c|}
\hline 424 & Sulindac & 2 & 0.57 & 1.39 & 2.72 & 3.16 & 200 & $\mathrm{~L}$ & $\mathrm{H}$ & 2 \\
\hline 425 & Sulpiride & 3 & 0.72 & 2.15 & 1.78 & 1.11 & 200 & $\mathrm{H}$ & $\mathrm{H}$ & 1 \\
\hline 426 & Tacrine & 1 & 0.23 & 0.76 & 2.3 & 3.27 & 200 & $\mathrm{~L}$ & $\mathrm{H}$ & 2 \\
\hline 427 & Tacrolimus & 2 & 0.71 & 3.98 & 3.98 & 5.78 & 5 & $\mathrm{H}$ & $\mathrm{H}$ & 1 \\
\hline 428 & Tadalafil & 2 & 0.31 & 2.27 & 3.27 & 2.58 & 20 & $\mathrm{~L}$ & $\mathrm{H}$ & 2 \\
\hline 429 & Talinolol & 3 & 0.81 & 2 & 1.97 & 3.15 & 100 & $\mathrm{H}$ & $\mathrm{H}$ & 1 \\
\hline 430 & Tamoxifen & 1 & 0 & 1.11 & 1.85 & 6.82 & 20 & $\mathrm{~L}$ & $\mathrm{H}$ & 2 \\
\hline 431 & Tamsulosin & 1 & 0.59 & 2.11 & 2.9 & 2.17 & 0.4 & $\mathrm{H}$ & $\mathrm{H}$ & 1 \\
\hline 432 & Telmisartan & 2 & 0.57 & 1.59 & 3.56 & 7.54 & 80 & $\mathrm{~L}$ & $\mathrm{H}$ & 2 \\
\hline 433 & Temazepam & 1 & 0.17 & 1.34 & 2.35 & 2.34 & 100 & $\mathrm{~L}$ & $\mathrm{H}$ & 2 \\
\hline 434 & Temozolomide & 2 & 0.49 & 1.82 & 2.49 & -0.81 & 250 & $\mathrm{H}$ & $\mathrm{L}$ & 3 \\
\hline 435 & Tenofovir disoproxil & 3 & 0.23 & 3.22 & 1.21 & 0.8 & 1000 & $\mathrm{H}$ & $\mathrm{H}$ & 1 \\
\hline 436 & Tenoxicam & 1 & 0.72 & 2.06 & 3.04 & 1.61 & 20 & $\mathrm{~L}$ & $\mathrm{H}$ & 2 \\
\hline 437 & Terbinafine & 2 & 0 & 0.86 & 1.34 & 5.96 & 250 & $\mathrm{~L}$ & $\mathrm{H}$ & 2 \\
\hline 438 & Terbutaline & 3 & 1.38 & 1.63 & 1.31 & 0.48 & 5 & $\mathrm{H}$ & $\mathrm{L}$ & 3 \\
\hline 439 & Terfenadine & 2 & 0.63 & 1.8 & 2.04 & 6.07 & 60 & $\mathrm{~L}$ & $\mathrm{H}$ & 2 \\
\hline 440 & Testolactone & 2 & 0 & 1.03 & 2.17 & 2.63 & 50 & $\mathrm{~L}$ & $\mathrm{H}$ & 2 \\
\hline 441 & Testosterone & 2 & 0.31 & 1.01 & 2.27 & 3.22 & 40 & $\mathrm{~L}$ & $\mathrm{H}$ & 2 \\
\hline 442 & Tetrabenazine & 2 & 0 & 1.44 & 2.02 & 3.81 & 25 & $\mathrm{~L}$ & $\mathrm{H}$ & 2 \\
\hline 443 & Thalidomide & 2 & 0.34 & 1.72 & 2.74 & 0.53 & 25 & $\mathrm{H}$ & $\mathrm{H}$ & 1 \\
\hline 444 & Theophylline & 1 & 0.35 & 1.29 & 1.99 & -0.03 & 600 & $\mathrm{H}$ & $\mathrm{H}$ & 1 \\
\hline 445 & Thioguanine & 1 & 0.77 & 1.14 & 1.47 & -1.7 & 40 & $\mathrm{H}$ & $\mathrm{L}$ & 3 \\
\hline 446 & Thioridazine & 1 & 0 & 1.13 & 1.93 & 6 & 200 & $\mathrm{~L}$ & $\mathrm{H}$ & 2 \\
\hline 447 & Thyroxine & 2 & 1.03 & 1.31 & 2.83 & 3.51 & 0.3 & $\mathrm{H}$ & $\mathrm{H}$ & 1 \\
\hline 448 & Tiaprofenic acid & 2 & 0.57 & 0.81 & 1.89 & 2.54 & 300 & $\mathrm{~L}$ & $\mathrm{H}$ & 2 \\
\hline 449 & Ticlopidine & 1 & 0 & 0.62 & 1.32 & 4.39 & 250 & $\mathrm{~L}$ & $\mathrm{H}$ & 2 \\
\hline 450 & Tilidine & 1 & 0 & 1.07 & 1.61 & 3.76 & 60 & $\mathrm{H}$ & $\mathrm{H}$ & 1 \\
\hline 451 & Tiludronic acid & 3 & 1.25 & 2.46 & 2.06 & 0.26 & 200 & $\mathrm{H}$ & $\mathrm{L}$ & 3 \\
\hline 452 & Tinidazole & 1 & 0 & 1.13 & 2.4 & -0.32 & 500 & $\mathrm{H}$ & $\mathrm{H}$ & 1 \\
\hline 453 & Tizanidine & 2 & 0.39 & 1.19 & 1.69 & 2.09 & 80 & $\mathrm{H}$ & $\mathrm{H}$ & 1 \\
\hline 454 & Tocainide & 3 & 0.47 & 1.24 & 1.62 & 0.26 & 600 & $\mathrm{H}$ & $\mathrm{H}$ & 1 \\
\hline 455 & Tolbutamide & 2 & 0.93 & 1.09 & 2.3 & 2.5 & 300 & $\mathrm{H}$ & $\mathrm{H}$ & 1 \\
\hline 456 & Tolcapone & 2 & 0.8 & 0.89 & 2.25 & 3.25 & 200 & $\mathrm{~L}$ & $\mathrm{H}$ & 2 \\
\hline 457 & Tolfenamic acid & 2 & 0.71 & 0.69 & 1.64 & 5.66 & 200 & $\mathrm{~L}$ & $\mathrm{H}$ & 2 \\
\hline 458 & Tolmetin & 2 & 0.57 & 0.97 & 1.93 & 2.21 & 600 & $\mathrm{H}$ & $\mathrm{H}$ & 1 \\
\hline 459 & Tolterodine & 1 & 0.5 & 1.08 & 1.42 & 5.24 & 2 & $\mathrm{H}$ & $\mathrm{H}$ & 1 \\
\hline 460 & Tolvaptan & 2 & 0.72 & 1.86 & 3.31 & 4.65 & 30 & $\mathrm{~L}$ & $\mathrm{H}$ & 2 \\
\hline 461 & Topiramate & 3 & 0.44 & 2.17 & 1.77 & 0.04 & 300 & $\mathrm{H}$ & $\mathrm{H}$ & 1 \\
\hline 462 & Topotecan & 3 & 0.67 & 2.73 & 3.69 & 0.73 & 1 & $\mathrm{H}$ & $\mathrm{H}$ & 1 \\
\hline
\end{tabular}


J Pharm Pharm Sci (www.cspsCanada.org) 22, 247 - 269, 2019

\begin{tabular}{|c|c|c|c|c|c|c|c|c|c|c|}
\hline 463 & Toremifene & 1 & 0 & 1.11 & 1.99 & 6.53 & 60 & $\mathrm{~L}$ & $\mathrm{H}$ & 2 \\
\hline 464 & Torsemide & 2 & 0.72 & 1.75 & 3.04 & 3.36 & 100 & $\mathrm{~L}$ & $\mathrm{H}$ & 2 \\
\hline 465 & Tramadol & 1 & 0.31 & 1.3 & 1.15 & 3.1 & 50 & $\mathrm{H}$ & $\mathrm{H}$ & 1 \\
\hline 466 & Trandolapril & 2 & 0.71 & 1.96 & 1.47 & 2.1 & 6 & $\mathrm{H}$ & $\mathrm{H}$ & 1 \\
\hline 467 & Tretinoin & 2 & 0.57 & 0.8 & 0.98 & 6.74 & 40 & $\mathrm{~L}$ & $\mathrm{H}$ & 2 \\
\hline 468 & Triamcinolone & 1 & 1.03 & 2.25 & 3.21 & 0.71 & 4 & $\mathrm{H}$ & $\mathrm{L}$ & 3 \\
\hline 469 & Triamcinolone acetonide & 1 & 0.56 & 2.14 & 3.13 & 2.21 & 4 & $\mathrm{H}$ & $\mathrm{H}$ & 1 \\
\hline 470 & Triamterene & 2 & 0.68 & 1.45 & 2.64 & 1.61 & 100 & $\mathrm{~L}$ & $\mathrm{H}$ & 2 \\
\hline 471 & Triclabendazole & 2 & 0.35 & 0.9 & 2.25 & 6.44 & 250 & $\mathrm{~L}$ & $\mathrm{H}$ & 2 \\
\hline 472 & Trifluoperazine & 1 & 0 & 1.42 & 1.79 & 4.69 & 10 & $\mathrm{~L}$ & $\mathrm{H}$ & 2 \\
\hline 473 & Trihexyphenidyl & 1 & 0.31 & 1.08 & 1.16 & 5.15 & 5 & $\mathrm{H}$ & $\mathrm{H}$ & 1 \\
\hline 474 & Trimetazidine & 3 & 0.16 & 1.67 & 2 & 1.18 & 20 & $\mathrm{H}$ & $\mathrm{H}$ & 1 \\
\hline 475 & Trimethoprim & 3 & 0.45 & 1.62 & 2.81 & 0.98 & 160 & $\mathrm{~L}$ & $\mathrm{H}$ & 2 \\
\hline 476 & Tropisetron & 1 & 0.31 & 1.21 & 1.78 & 2.88 & 5 & $\mathrm{H}$ & $\mathrm{H}$ & 1 \\
\hline 477 & Trospium & 3 & 0.17 & 1.1 & 1.57 & -1.16 & 20 & $\mathrm{H}$ & $\mathrm{H}$ & 1 \\
\hline 478 & Ursodiol & 2 & 1.2 & 1.35 & 2.4 & 4.51 & 500 & $\mathrm{~L}$ & $\mathrm{H}$ & 2 \\
\hline 479 & Valacyclovir & 1 & 0.63 & 2.75 & 2.44 & -1.22 & 1000 & $\mathrm{H}$ & $\mathrm{L}$ & 3 \\
\hline 480 & Valdecoxib & 2 & 0.44 & 1.24 & 2.5 & 1.83 & 20 & $\mathrm{~L}$ & $\mathrm{H}$ & 2 \\
\hline 481 & Valganciclovir & 1 & 0.86 & 3.15 & 2.64 & -2.18 & 450 & $\mathrm{H}$ & $\mathrm{L}$ & 3 \\
\hline 482 & Valproic Acid & 1 & 0.61 & 0.46 & 0.54 & 2.76 & 250 & $\mathrm{H}$ & $\mathrm{H}$ & 1 \\
\hline 483 & Valsartan & 4 & 1.21 & 1.82 & 3.32 & 4.86 & 320 & $\mathrm{~L}$ & $\mathrm{H}$ & 2 \\
\hline 484 & Vardenafil & 1 & 0.13 & 2.97 & 1.26 & 2.23 & 100 & $\mathrm{H}$ & $\mathrm{H}$ & 1 \\
\hline 485 & Vigabatrin & 3 & 0.78 & 0.99 & 0.99 & -2.22 & 500 & $\mathrm{H}$ & $\mathrm{L}$ & 3 \\
\hline 486 & Vitamin A (Retinol) & 2 & 0.31 & 0.75 & 0.81 & 6.4 & 110 & $\mathrm{H}$ & $\mathrm{H}$ & 1 \\
\hline 487 & Vitamin $B_{1}$ & 3 & 0.54 & 1.04 & 1.58 & -5.97 & 500 & $\mathrm{H}$ & $\mathrm{L}$ & 3 \\
\hline 488 & Vitamin B2 & 4 & 1.33 & 2.69 & 2.71 & -0.73 & 100 & $\mathrm{H}$ & $\mathrm{L}$ & 3 \\
\hline 489 & Vitamin D3 & 1 & 0.31 & 0.71 & 0.75 & 9.48 & 1.4 & $\mathrm{H}$ & $\mathrm{H}$ & 1 \\
\hline 490 & Voriconazole & 2 & 0.31 & 1.5 & 2.19 & 0.52 & 200 & $\mathrm{H}$ & $\mathrm{H}$ & 1 \\
\hline 491 & Zafirlukast & 2 & 0.85 & 2.13 & 4.09 & 7.09 & 20 & $\mathrm{~L}$ & $\mathrm{H}$ & 2 \\
\hline 492 & Zidovudine & 1 & 0.47 & 1.7 & 2.03 & 0.04 & 200 & $\mathrm{H}$ & $\mathrm{H}$ & 1 \\
\hline 493 & Zileuton & 2 & 0.71 & 1.15 & 2.12 & 2.48 & 600 & $\mathrm{~L}$ & $\mathrm{H}$ & 2 \\
\hline 494 & Zolmitriptan & 1 & 0.48 & 1.65 & 2.67 & 1.29 & 5 & $\mathrm{H}$ & $\mathrm{H}$ & 1 \\
\hline 495 & Zonisamide & 1 & 0.44 & 1.11 & 1.95 & -0.36 & 100 & $\mathrm{H}$ & $\mathrm{H}$ & 1 \\
\hline 496 & Zopiclone & 1 & 0 & 2.43 & 3.2 & 1.25 & 7.5 & $\mathrm{H}$ & $\mathrm{H}$ & 1 \\
\hline \multicolumn{11}{|c|}{ Test set } \\
\hline 1 & Acitretin & 2 & 0.57 & 0.97 & 1.32 & 6.07 & 25 & $\mathrm{~L}$ & $\mathrm{H}$ & 2 \\
\hline 2 & Alfuzosin & 1 & 0.48 & 2.24 & 3.39 & 2.55 & 10 & $\mathrm{~L}$ & $\mathrm{H}$ & 2 \\
\hline 3 & Aliskiren & 1 & 1.2 & 3.06 & 3.65 & 3.51 & 300 & $\mathrm{~L}$ & $\mathrm{H}$ & 2 \\
\hline 4 & Aminocaproic acid & 3 & 0.78 & 0.91 & 0.95 & -2.24 & 1000 & $\mathrm{H}$ & $\mathrm{L}$ & 3 \\
\hline
\end{tabular}


J Pharm Pharm Sci (www.cspsCanada.org) 22, 247 - 269, 2019

\begin{tabular}{|c|c|c|c|c|c|c|c|c|c|c|}
\hline 5 & Amprenavir & 2 & 0.64 & 2.61 & 3.52 & 3.29 & 50 & $\mathrm{~L}$ & $\mathrm{H}$ & 2 \\
\hline 6 & Armodafinil & 2 & 0.49 & 1.47 & 3.2 & 0.94 & 250 & $\mathrm{~L}$ & $\mathrm{H}$ & 2 \\
\hline 7 & Aspirin & 1 & 0.57 & 0.77 & 1.42 & 1.02 & 500 & $\mathrm{H}$ & $\mathrm{H}$ & 1 \\
\hline 8 & Auranofin & 4 & 0 & 1.82 & 2.17 & 3.79 & 3 & $\mathrm{H}$ & $\mathrm{H}$ & 1 \\
\hline 9 & Azapropazone & 4 & 0 & 2.16 & 2.1 & 1.79 & 300 & $\mathrm{H}$ & $\mathrm{H}$ & 1 \\
\hline 10 & Baclofen & 3 & 0.78 & 1.02 & 1.47 & -0.62 & 20 & $\mathrm{H}$ & $\mathrm{L}$ & 3 \\
\hline 11 & Betaxolol & 1 & 0.29 & 1.53 & 1.31 & 2.32 & 20 & $\mathrm{H}$ & $\mathrm{H}$ & 1 \\
\hline 12 & Bevantolol & 2 & 0.29 & 1.82 & 2.14 & 3 & 200 & $\mathrm{H}$ & $\mathrm{H}$ & 1 \\
\hline 13 & Bezafibrate & 2 & 0.83 & 1.35 & 2.54 & 3.7 & 200 & $\mathrm{~L}$ & $\mathrm{H}$ & 2 \\
\hline 14 & Bromazepam & 1 & 0.47 & 1.27 & 1.93 & 1.7 & 6 & $\mathrm{H}$ & $\mathrm{H}$ & 1 \\
\hline 15 & Bromocriptine & 1 & 0.79 & 3.66 & 4.28 & 6.58 & 5 & $\mathrm{H}$ & $\mathrm{H}$ & 1 \\
\hline 16 & Capecitabine & 1 & 0.6 & 2.4 & 2.41 & 0.84 & 500 & $\mathrm{H}$ & $\mathrm{H}$ & 1 \\
\hline 17 & Caprylidene & 1 & 0 & 1.12 & 1.45 & 9.97 & 2000 & $\mathrm{~L}$ & $\mathrm{H}$ & 2 \\
\hline 18 & Carvedilol & 2 & 0.62 & 2.09 & 3 & 4.04 & 25 & $\mathrm{~L}$ & $\mathrm{H}$ & 2 \\
\hline 19 & Cefixime & 4 & 1.64 & 3.12 & 4.01 & 0.25 & 400 & $\mathrm{~L}$ & $\mathrm{~L}$ & 4 \\
\hline 20 & Celecoxib & 2 & 0.44 & 1.22 & 2.43 & 4.37 & 200 & $\mathrm{~L}$ & $\mathrm{H}$ & 2 \\
\hline 21 & Celiprolol & 3 & 0.61 & 2.35 & 2.34 & 1.86 & 200 & $\mathrm{H}$ & $\mathrm{H}$ & 1 \\
\hline 22 & Cephalexin & 3 & 1.06 & 2.54 & 3.27 & -1.84 & 750 & $\mathrm{H}$ & $\mathrm{L}$ & 3 \\
\hline 23 & Cevimeline & 1 & 0 & 0.9 & 0.86 & 1.14 & 30 & $\mathrm{H}$ & $\mathrm{H}$ & 1 \\
\hline 24 & Chloral hydrate & 1 & 0.75 & 0.59 & 0.97 & 0.72 & 500 & $\mathrm{H}$ & $\mathrm{H}$ & 1 \\
\hline 25 & Chloramphenicol & 1 & 0.87 & 1.65 & 2.66 & 1.28 & 250 & $\mathrm{~L}$ & $\mathrm{H}$ & 2 \\
\hline 26 & Cinacalcet & 2 & 0.13 & 0.63 & 1.37 & 6.35 & 90 & $\mathrm{~L}$ & $\mathrm{H}$ & 2 \\
\hline 27 & Ciprofloxacin & 4 & 0.73 & 1.85 & 2.5 & -0.73 & 750 & $\mathrm{H}$ & $\mathrm{L}$ & 3 \\
\hline 28 & Clobazam & 1 & 0 & 1.47 & 2.49 & 2.44 & 10 & $\mathrm{~L}$ & $\mathrm{H}$ & 2 \\
\hline 29 & Clomipramine & 1 & 0 & 0.89 & 1.66 & 5.92 & 75 & $\mathrm{~L}$ & $\mathrm{H}$ & 2 \\
\hline 30 & Clonidine & 3 & 0.39 & 0.9 & 1.19 & 1.73 & 0.3 & $\mathrm{H}$ & $\mathrm{H}$ & 1 \\
\hline 31 & Clorazepate & 1 & 1.04 & 1.34 & 2.14 & 2.51 & 15 & $\mathrm{~L}$ & $\mathrm{H}$ & 2 \\
\hline 32 & Clotrimazole & 2 & 0 & 0.78 & 2.37 & 5.25 & 10 & $\mathrm{~L}$ & $\mathrm{H}$ & 2 \\
\hline 33 & Cyclobenzaprine & 1 & 0 & 0.83 & 1.41 & 5.1 & 10 & $\mathrm{~L}$ & $\mathrm{H}$ & 2 \\
\hline 34 & Cyclophosphamide & 1 & 0.14 & 1.18 & 2.2 & 0.8 & 50 & $\mathrm{H}$ & $\mathrm{H}$ & 1 \\
\hline 35 & Dapsone & 2 & 0.45 & 1.35 & 2.84 & 0.89 & 100 & $\mathrm{~L}$ & $\mathrm{H}$ & 2 \\
\hline 36 & Desmopressin & 3 & 4.3 & 8.13 & 11.82 & -3.14 & 0.2 & $\mathrm{H}$ & $\mathrm{L}$ & 3 \\
\hline 37 & Desvenlafaxine & 3 & 0.81 & 1.22 & 1.34 & 2.68 & 100 & $\mathrm{H}$ & $\mathrm{H}$ & 1 \\
\hline 38 & Domperidone & 2 & 0.72 & 1.83 & 3.13 & 4.27 & 20 & $\mathrm{~L}$ & $\mathrm{H}$ & 2 \\
\hline 39 & Ebastine & 2 & 0 & 1.41 & 2.37 & 6.94 & 10 & $\mathrm{~L}$ & $\mathrm{H}$ & 2 \\
\hline 40 & Entecavir & 3 & 1.05 & 2.29 & 2.18 & -2.58 & 1 & $\mathrm{H}$ & $\mathrm{L}$ & 3 \\
\hline 41 & Ergotamine tartrate & 1 & 0.79 & 3.69 & 4.6 & 4.66 & 2 & $\mathrm{H}$ & $\mathrm{H}$ & 1 \\
\hline 42 & Erythromycin stearate & 4 & 0.91 & 4.49 & 3.3 & 1.61 & 500 & $\mathrm{H}$ & $\mathrm{H}$ & 1 \\
\hline 43 & Escitalopram & 1 & 0 & 1.08 & 1.87 & 3.13 & 20 & $\mathrm{~L}$ & $\mathrm{H}$ & 2 \\
\hline
\end{tabular}


J Pharm Pharm Sci (www.cspsCanada.org) 22, 247 - 269, 2019

\begin{tabular}{|c|c|c|c|c|c|c|c|c|c|c|}
\hline 44 & Etoposide & 3 & 0.6 & 3.23 & 4.11 & 0.03 & 50 & $\mathrm{~L}$ & $\mathrm{~L}$ & 4 \\
\hline 45 & Ezetimibe & 2 & 0.81 & 1.77 & 2.61 & 3.96 & 10 & $\mathrm{~L}$ & $\mathrm{H}$ & 2 \\
\hline 46 & Famotidine & 3 & 1.21 & 2.78 & 2.24 & -1.17 & 40 & $\mathrm{H}$ & $\mathrm{L}$ & 3 \\
\hline 47 & Febuxostat & 2 & 0.57 & 1.11 & 2.25 & 4.4 & 80 & $\mathrm{~L}$ & $\mathrm{H}$ & 2 \\
\hline 48 & Fexofenadine & 3 & 1.2 & 2.12 & 2.48 & 1.96 & 180 & $\mathrm{H}$ & $\mathrm{L}$ & 3 \\
\hline 49 & Fosfomycin tromethamine & 3 & 0.63 & 1.38 & 0.98 & -0.23 & 3000 & $\mathrm{H}$ & $\mathrm{L}$ & 3 \\
\hline 50 & Idebenone & 2 & 0.31 & 1.56 & 0.84 & 3.42 & 180 & $\mathrm{H}$ & $\mathrm{H}$ & 1 \\
\hline 51 & Indapamide & 1 & 0.7 & 1.86 & 3.2 & 2.96 & 2.5 & $\mathrm{H}$ & $\mathrm{H}$ & 1 \\
\hline 52 & Indoramin & 2 & 0.57 & 1.49 & 2.66 & 2.84 & 25 & $\mathrm{~L}$ & $\mathrm{H}$ & 2 \\
\hline 53 & Iopanoic acid & 4 & 0.85 & 0.74 & 2 & 4.7 & 500 & $\mathrm{~L}$ & $\mathrm{H}$ & 2 \\
\hline 54 & Isradipine & 2 & 0.13 & 1.79 & 2.15 & 3.92 & 5 & $\mathrm{H}$ & $\mathrm{H}$ & 1 \\
\hline 55 & Ivermectin & 1 & 0.68 & 4.23 & 3.21 & 5.39 & 3 & $\mathrm{H}$ & $\mathrm{H}$ & 1 \\
\hline 56 & Ketoprofen & 2 & 0.57 & 0.87 & 1.97 & 2.76 & 75 & $\mathrm{~L}$ & $\mathrm{H}$ & 2 \\
\hline 57 & Levofloxacin & 3 & 0.57 & 2.05 & 2.58 & -0.51 & 750 & $\mathrm{H}$ & $\mathrm{L}$ & 3 \\
\hline 58 & Lisinopril & 3 & 1.49 & 2.47 & 2.98 & -1.69 & 40 & $\mathrm{H}$ & $\mathrm{L}$ & 3 \\
\hline 59 & Lofepramine & 2 & 0 & 1.35 & 2.63 & 7.29 & 70 & $\mathrm{~L}$ & $\mathrm{H}$ & 2 \\
\hline 60 & Lomefloxacin & 3 & 0.73 & 1.81 & 2.37 & -0.11 & 400 & $\mathrm{H}$ & $\mathrm{L}$ & 3 \\
\hline 61 & Maraviroc & 1 & 0.26 & 1.75 & 2.62 & 3.26 & 300 & $\mathrm{~L}$ & $\mathrm{H}$ & 2 \\
\hline 62 & Memantine & 3 & 0.21 & 0.66 & 0.58 & 3.03 & 10 & $\mathrm{H}$ & $\mathrm{H}$ & 1 \\
\hline 63 & Mesna & 1 & 0.31 & 0.92 & 1.52 & -1.55 & 400 & $\mathrm{H}$ & $\mathrm{L}$ & 3 \\
\hline 64 & Methadone & 1 & 0 & 1.09 & 1.72 & 4.17 & 10 & $\mathrm{~L}$ & $\mathrm{H}$ & 2 \\
\hline 65 & Methaqualone & 2 & 0 & 0.94 & 1.74 & 3.65 & 500 & $\mathrm{~L}$ & $\mathrm{H}$ & 2 \\
\hline 66 & Metoclopramide & 3 & 0.5 & 1.63 & 2.31 & 2.23 & 10 & $\mathrm{H}$ & $\mathrm{H}$ & 1 \\
\hline 67 & Naloxone & 1 & 0.5 & 1.75 & 2.02 & 0.16 & 2 & $\mathrm{H}$ & $\mathrm{H}$ & 1 \\
\hline 68 & Naltrexone & 1 & 0.5 & 1.71 & 2.03 & 0.36 & 100 & $\mathrm{H}$ & $\mathrm{H}$ & 1 \\
\hline 69 & Nitrofurantoin & 4 & 0.24 & 1.34 & 2.03 & -0.47 & 100 & $\mathrm{H}$ & $\mathrm{H}$ & 1 \\
\hline 70 & Nitroglycerin & 1 & 0 & 0.45 & 1.87 & 1.76 & 0.4 & $\mathrm{H}$ & $\mathrm{H}$ & 1 \\
\hline 71 & Omeprazole & 1 & 0.35 & 2.05 & 3.18 & 2.57 & 40 & $\mathrm{~L}$ & $\mathrm{H}$ & 2 \\
\hline 72 & Oxatomide & 2 & 0.33 & 1.89 & 2.85 & 5.62 & 30 & $\mathrm{~L}$ & $\mathrm{H}$ & 2 \\
\hline 73 & p-Aminosalicylic acid & 1 & 0.93 & 0.65 & 1.48 & 1.06 & 1200 & $\mathrm{H}$ & $\mathrm{L}$ & 3 \\
\hline 74 & Phenylpropanolamine & 3 & 0.46 & 1.22 & 1.09 & 0.58 & 75 & $\mathrm{H}$ & $\mathrm{H}$ & 1 \\
\hline 75 & Pitavastatin & 2 & 1.2 & 1.59 & 2.63 & 3.59 & 4 & $\mathrm{H}$ & $\mathrm{H}$ & 1 \\
\hline 76 & Pravastatin & 3 & 1.51 & 1.89 & 2.11 & 2.05 & 80 & $\mathrm{H}$ & $\mathrm{H}$ & 1 \\
\hline 77 & Praziquantel & 2 & 0 & 1.46 & 2.42 & 3.36 & 600 & $\mathrm{~L}$ & $\mathrm{H}$ & 2 \\
\hline 78 & Pregabalin & 3 & 0.78 & 0.97 & 0.93 & -0.92 & 300 & $\mathrm{H}$ & $\mathrm{L}$ & 3 \\
\hline 79 & Quazepam & 2 & 0 & 0.49 & 1.57 & 3.2 & 15 & $\mathrm{~L}$ & $\mathrm{H}$ & 2 \\
\hline 80 & Ramipril & 1 & 0.71 & 1.96 & 2.68 & 1.54 & 10 & $\mathrm{H}$ & $\mathrm{H}$ & 1 \\
\hline 81 & Ridogrel & 1 & 0.57 & 1.08 & 1.49 & 4.54 & 5 & $\mathrm{H}$ & $\mathrm{H}$ & 1 \\
\hline 82 & Ritodrine & 3 & 1.38 & 1.76 & 1.83 & 1.65 & 10 & $\mathrm{H}$ & $\mathrm{L}$ & 3 \\
\hline
\end{tabular}


J Pharm Pharm Sci (www.cspsCanada.org) 22, 247 - 269, 2019

\begin{tabular}{|c|c|c|c|c|c|c|c|c|c|c|}
\hline 83 & Ritonavir & 2 & 0.88 & 3.14 & 5.05 & 4.94 & 100 & $\mathrm{~L}$ & $\mathrm{H}$ & 2 \\
\hline 84 & Rolitetracycline & 3 & 1.86 & 4.09 & 4.02 & 0.47 & 250 & $\mathrm{H}$ & $\mathrm{L}$ & 3 \\
\hline 85 & Rufinamide & 2 & 0.49 & 1.12 & 2.18 & 0.51 & 400 & $\mathrm{H}$ & $\mathrm{H}$ & 1 \\
\hline 86 & Sulfadiazine & 4 & 0.59 & 1.4 & 2.58 & 0.1 & 500 & $\mathrm{H}$ & $\mathrm{H}$ & 1 \\
\hline 87 & Telithromycin & 2 & 0.12 & 4.4 & 4.53 & 3.75 & 400 & $\mathrm{~L}$ & $\mathrm{H}$ & 2 \\
\hline 88 & Temocapril & 1 & 0.71 & 2.18 & 2.96 & 2.1 & 4 & $\mathrm{H}$ & $\mathrm{H}$ & 1 \\
\hline 89 & Terazosin & 1 & 0.23 & 2.27 & 3.49 & 2.18 & 10 & $\mathrm{~L}$ & $\mathrm{H}$ & 2 \\
\hline 90 & Thiabendazole & 2 & 0.35 & 0.72 & 1.94 & 2.36 & 500 & $\mathrm{~L}$ & $\mathrm{H}$ & 2 \\
\hline 91 & Timolol & 1 & 0.29 & 1.91 & 1.68 & 1.21 & 20 & $\mathrm{H}$ & $\mathrm{H}$ & 1 \\
\hline 92 & Tipranavir & 2 & 0.67 & 1.86 & 3.08 & 7.76 & 500 & $\mathrm{~L}$ & $\mathrm{H}$ & 2 \\
\hline 93 & Tolazamide & 2 & 0.59 & 1.62 & 2.49 & 1.34 & 500 & $\mathrm{H}$ & $\mathrm{H}$ & 1 \\
\hline 94 & Trazodone & 2 & 0 & 1.92 & 2.47 & 3.85 & 300 & $\mathrm{~L}$ & $\mathrm{H}$ & 2 \\
\hline 95 & Triazolam & 1 & 0 & 0.83 & 2.03 & 2.62 & 4 & $\mathrm{H}$ & $\mathrm{H}$ & 1 \\
\hline 96 & Vitamin B6 & 1 & 0.94 & 1.5 & 1.27 & -0.35 & 25 & $\mathrm{H}$ & $\mathrm{L}$ & 3 \\
\hline 97 & Warfarin & 2 & 0.31 & 1.23 & 2.28 & 2.9 & 10 & $\mathrm{~L}$ & $\mathrm{H}$ & 2 \\
\hline 98 & Zalcitabine & 3 & 0.44 & 1.9 & 1.78 & -1.25 & 0.75 & $\mathrm{H}$ & $\mathrm{L}$ & 3 \\
\hline 99 & Zaleplon & 2 & 0 & 1.42 & 2.6 & 1.44 & 10 & $\mathrm{~L}$ & $\mathrm{H}$ & 2 \\
\hline
\end{tabular}

Table 3. BDDCS class changes from initial publication for nine drugs and prediction of BDDCS class in this study

\begin{tabular}{|c|c|c|c|}
\hline Generic Name & Listed class $^{\mathbf{a}}$ & Updated class $^{b}$ & Prediction of BDDCS \\
\hline Aliskiren & 1 & 3 & 2 \\
\hline Clonidine & 3 & 1 & 1 \\
\hline Colchicine & 1 & 3 & 1 \\
\hline Diclofenac & 1 & 2 & 2 \\
\hline Flecainide & 3 & 1 & 1 \\
\hline Metoclopramide & 3 & 1 & 1 \\
\hline Pindolol & 3 & 1 & 1 \\
\hline Pitavastatin & 2 & 4 & 1 \\
\hline Saxagliptin & 3 & 1 & 2 \\
\hline
\end{tabular}

$$
{ }^{\text {aRef. [8] }}
$$

${ }^{\text {bRef. [34] }}$ 